\title{
Probabilistic, Iterated and Quantum-Iterated Computational Methods in Gray-Level Image Restoration
}

\author{
Kazuyuki TANAKA* and Tsuyoshi HORIGUCHI \\ Department of Computer and Mathematical Sciences, Graduate School of Information Sciences, \\ Tohoku University, Sendai 980-8579, Japan
}

Received December 13, 2000; final version accepted April 23, 2001

\begin{abstract}
For image restoration as an energy minimization problem, an energy function is expressed by using a hamiltonian of a classical spin system only with finite range interactions. The problem is formulated in terms of a probabilistic model with a Gibbs distribution and hence may be regarded as a Markov random field model. We compare the results obtained by proposed methods with those obtained by the probabilistic computational algorithms for restoration of natural images within a framework of the mean-field approximation or the pair approximation. We clarify a role of Nishimori-Wong's inequality in the Markov random field model as for the practical algorithms to the image restoration of natural images through some numerical experiments.
\end{abstract}

KEYWORDS: Bayes statistics, probabilitic algorithm, quantum computing, Markov random fields, image restoration

\section{Introduction}

Recently, from standpoints of the computer sciences, the statistics and the statistical mechanics, many authors have shown an interest in image restoration using the Markov random field (MRF) models, in which a configuration of pixel values depends only on values at its nearest neighbour pixels [1]. The MRF model is constructed under assumptions for a priori probability distribution of original images, for degradation process from an original image to a degraded image using the Bayes formula and for the Gibbs distribution with an energy function. The obtained probability distribution is called a posteriori probability distribution. The energy function is expressed in terms of variables for pixels and interaction parameters between the degraded image and the original image. In the statistics, both the temperature and the interaction parameters are called hyperparameters. Besag [2] and Lakshmanan and Derin [3] gave a framework of hyperparameter estimation based on the maximum likelihood estimation. The maximum likelihood estimation in the MRF model was extended to the expectation maximization algorithm [4] or the maximum marginal likelihood estimation [5]. Although the above construction scheme of the MRF model is based on statistics, the practical algorithms for determination of hyperparameters have often been constructed by use of the statistical-mechanical techniques. It is interesting to reformulate the framework of hyperparameter estimation based on the maximum likelihood estimation from a standpoint of statistical mechanics.

In the Bayes analysis, the restored image is obtained by maximizing the a posteriori probability distribution and this framework is called a maximum a posteriori (MAP) estimation. On the other hand, the restored image can also be obtained by maximizing the posterior marginal probability distribution for each pixel and this framework is called a maximum posterior marginal (MPM) estimation [5]. Marroquin et al. [6] suggested that the MPM estimation gives a restored image which is closer to the original image than that obtained by the MAP estimation through some numerical experiments. Though the MAP estimation corresponds to obtaining of the ground state configuration by using the temperature as a role of just control parameter of annealing procedures, the MAP estimation gives a restored image which depends on the scheduling temperature. Recently, Nishimori and Wong [7] have studied the suggestion given by Marroquin et al. from a standpoint of spin glass theory. They have exactly calculated the statistical average of Hamming distances between original images and their corresponding restored images as for binary images by using the replica method under the a priori probability distribution. Carlucci and Inoue [8] have extended the investigation of the binary images to a case of gray-level images by using the replica method and also by Monte Carlo simulations; they have used the Gibbs distribution for a chiral Potts model. Although the investigations show that the MPM estimation is better than the MAP estimation, the difference between them is about $3 \%$ as shown by some exact estimations of performance and by numerical experiments [7], [8]. This means that we do not need to reduce the temperature to a sufficiently small positive value in the annealing procedure and that it is sufficient if the temperature is reduced to the value corre-

* CORRESPONDENCE: Kazuyuki TANAKA, Department of Computer and Mathematical Sciences, Graduate School of Information Sciences, Tohoku University, Aramaki-aza-aoba 04, Aoba-ku, Sendai 980-8579, Japan. Phone: +81-22-217-5899, FAX: +81-22-217-5851, E-mail: kazu@statp.is.tohoku.ac.jp 
sponding to the degree of degradation; we have to estimate the restored image by using the posterior marginal probability distribution at the value of temperature corresponding to the degradation point.

In the search for optimal solutions of MRF model, many authors applied the iterated conditional modes (ICM) algorithm [2]. Although the ICM algorithm is a simple algorithm and can erase noise when the noise is at isolated pixels, it is difficult to avoid local minima and hence it cannot erase the noise when two or more adjoint pixels are affected by the noise. In the statistical mechanics, we have some useful fluctuations to avoid local minima. One of them is a thermal fluctuation. In order to adopt the thermal fluctuation effect as annealing procedure, we introduce temperature in the form of Gibbs distribution. Geiger and Girosi [13], and Zhang [4], [14] proposed deterministic algorithms, called the mean field annealing (MFA) algorithm, which is based on the mean-field approximation. On the other hand, Morita [15] gave a general methodology of construction of higher-order approximation in statistical mechanics, which is called cluster variation method (CVM). Tanaka and Morita [16] proposed another deterministic algorithm, called the pair annealing (PA) algorithm, which is based on the pair approximation in the CVM. They gave a simple example of its application to the MRF model in Ref. [16]. In the standpoint of the CVM, Morita and Tanaka [18] proposed a method of the extension of the ICM to a cluster type algorithm, called the cluster zero-temperature process (CZTP), which is obtained as the zero-temperature limit in the optimization algorithm of the CVM. The other important fluctuation to avoid local minima is a quantum fluctuation. We introduced a quantum MRF model and constructed a quantum mean-field iteration (QMFI) algorithm by using the mean-field approximation [19]. Inoue [20], [21] has also studied about the statistical performance of image restorations by means of the quantum MRF model from a standpoint of spin glass theory.

Hence, it is interesting to clarify which effect is the most important to avoid local minima, the effect of the size of local cluster, the effect of the thermal fluctuation or another effect such as quantum fluctuation. If the statistical fluctuations are important, we expect that the QMFI algorithm as well as the MFA algorithm will give better results. If the local cluster size is important, the CVM will give better results. If the thermal fluctuation, particularly near the values of hyperparameters specified by Nishimori-Wong's inequality in the MRF model as mentioned above, is the most important, the MFA and the PA algorithm will give the better results.

One of the main purposes of the present paper is to extend the formulation of the QMFI algorithm given for images with 2 grades, say black and white, to images with $q$ grades, when $q>2$. The other purpose is to clarify how Nishimori-Wong's inequality for image restorations in the MRF model plays an important role to the MFA and the PA algorithm for image restoration of natural images. It can be proved statistically that the optimal values of hyperparameters obtained from Nishimori-Wong's inequality are equivalent to those by the maximum likelihood estimates. The practical algorithms for calculation of the maximum likelihood estimates are given by using the CVM. In searching the optimal configurations of the MRF models defined at the obtained maximum likelihood estimates of hyperparameters, we adopt the MFA and the CVM algorithm as those in which the iteration processes pass through near the maximum likelihood estimates. On the other hand, we adopt the ICM, the CZTP and the QMFI algorithm by which the hyperparameters do not pass through near their values by the maximum likelihood estimate in these iteration processes. For these algorithms, some numerical experiments are given for image restorations to natural images with 8 grades and 256 grades. The a priori probability distributions of the MRF model in the present paper are constructed by modifying the Potts model.

The present paper is organized in the following way. In Sec. 2, we explain the energy function and the probabilistic model constructed within a framework of the Bayes statistics and the maximum likelihood estimation. In Sec. 3, we give some probabilistic computational algorithms for the search of minimum-energy configuration and the hyperparameter estimation by using the mean-field and the pair approximation. In Sec. 4, we explain briefly the ICM algorithm given in Ref. [2] and the CZTP algorithm given in Ref. [18]. In Sec. 5, we extend the QMFI algorithm for images with 2 grades given in Ref. [19] to that for gray-level images. In Sec. 6, we give a practical algorithm based on the formalism for the MRF model, which is given for image restorations of natural images with 256 grades in Sec. 2. In Sec. 7, we give the results by numerical experiments. Some concluding remarks are given in Sec. 8.

\section{Markov Random Field Model}

In this section, we give an energy function for the image restoration of natural images within a framework of the Bayes statistics and the maximum likelihood estimation. We consider a digital image with $q$ grades on the $M$ $\times N$ finite square lattice

$$
\mathbf{I} \equiv\{(i, j) \mid i=1,2, \cdots, M, j=1,2, \cdots, N\} .
$$

We express the random fields of an original and a degraded image by $\mathbf{X} \equiv\left\{X_{i, j} \mid(i, j) \in \mathbf{I}\right\}$ and $\mathbf{Y} \equiv\left\{Y_{i, j} \mid(i, j) \in\right.$ $\mathbf{I}\}$, respectively. The random variables $X_{i, j}$ and $Y_{i, j}$ take values in $\mathbf{Q} \equiv\{0,1,2, \cdots, q-1\}$. For a degradation process, we assume that a degraded image $\mathbf{y} \equiv\left\{\boldsymbol{y}_{i, j} \mid(i, j) \in \mathbf{I}\right\}$ is obtained from the original image $\mathbf{x}^{*} \equiv$ $\left\{x_{i, j}^{*} \mid(i, j) \in \mathbf{I}\right\}$ by changing a value at each pixel to another value by a probability $p^{*}$, independently of values at 
the other pixels. The conditional probability $\operatorname{Pr}\left\{\mathbf{Y}=\mathbf{y} \mid \mathbf{X}=\mathbf{x}^{*}, p^{*}\right\}$ for the degraded image $\mathbf{y}$ when the original image is $\mathbf{x}^{*}$ is given by

$$
\begin{aligned}
\operatorname{Pr}\left\{\mathbf{Y}=\mathbf{y} \mid \mathbf{X}=\mathbf{x}^{*}, p^{*}\right\} & \equiv \prod_{(i, j) \in \mathbf{I}}\left\{\left(1-\delta\left(x_{i, j}^{*}, y_{i, j}\right)\right) p^{*}+\left(1-(q-1) p^{*}\right) \delta\left(x_{i, j}^{*}, y_{i, j}\right)\right\} \\
& =\frac{\exp \left(-\frac{1}{T\left(p^{*}\right)} d\left(\mathbf{x}^{*}, \mathbf{y}\right)\right)}{\sum_{\mathbf{y} \in \mathbf{Q}^{u N}} \exp \left(-\frac{1}{T\left(p^{*}\right)} d\left(\mathbf{x}^{*}, \mathbf{y}\right)\right)},
\end{aligned}
$$

where

$$
d\left(\mathbf{x}^{*}, \mathbf{y}\right) \equiv \sum_{(i, j) \in \mathbf{I}}\left(1-\delta\left(x_{i, j}^{*}, y_{i, j}\right)\right), \quad T\left(p^{*}\right) \equiv \frac{1}{\ln \left(\frac{1-(q-1) p^{*}}{p^{*}}\right)} .
$$

Here, $\delta(a, b)$ is Kronecker's delta $\delta_{a, b}$. In the present paper, we consider only $T\left(p^{*}\right)>0$ and hence $p^{*}<1 / q$.

We assume that the original image is one of images which are produced by the $a$ priori probability distribution $\operatorname{Pr}\{\mathbf{X}=\mathbf{x}\}$ defined by

$$
\begin{aligned}
\operatorname{Pr}\{\mathbf{X}=\mathbf{x}\} & =\operatorname{Pr}\left\{\mathbf{X}=\mathbf{x} \mid \mathbf{K}^{*}\right\} \\
& \equiv \frac{\exp \left(-K_{k}^{*} \sum_{n=k}^{q-1} \sigma_{n}(\mathbf{x})-\sum_{n=1}^{k-1} K_{n}^{*} \sigma_{n}(\mathbf{x})\right)}{\sum_{\mathbf{x} \in \mathbf{Q}^{M v}} \exp \left(-K_{k}^{*} \sum_{n=k}^{q-1} \sigma_{n}(\mathbf{x})-\Sigma_{n=1}^{k-1} K_{n}^{*} \sigma_{n}(\mathbf{x})\right)},
\end{aligned}
$$

where $\mathbf{K}^{*} \equiv\left\{K_{n}^{*} \mid n=1,2, \cdots, k\right\}$ is a set of hyperparameters and $\sigma_{n}(\mathbf{x})$ is defined by

$$
\begin{array}{r}
\sigma_{n}(\mathbf{x}) \equiv \sum_{(i, j) \in \mathrm{I}}\left(\delta\left(\left|x_{i, j}-x_{i+1, j}\right|, n\right)+\delta\left(\left|x_{i, j}-x_{i, j+1}\right|, n\right)\right) \\
(n=0,1,2, \cdots, q-1) .
\end{array}
$$

Since we have $\sum_{n=0}^{q-1} \sigma_{n}(\mathbf{x})=2 M N$, we notice that the quantity $\sigma_{0}(\mathbf{x})$ can be expressed in terms of other quantities $\sigma_{n}(\mathbf{x})(k=1,2, \cdots, k-1)$. In the Bayes formula, the a posteriori probability distribution $\operatorname{Pr}\left\{\mathbf{X}=\mathbf{x} \mid \mathbf{Y}=\mathbf{y}^{*}\right\}$ for the original image $\mathbf{x} \equiv\left\{x_{i, j} \mid(i, j) \in \mathbf{I}\right\}$ when the degraded image is $\mathbf{y}^{*}$ is expressed as

$$
\begin{aligned}
\operatorname{Pr}\left\{\mathbf{X}=\mathbf{x} \mid \mathbf{Y}=\mathbf{y}^{*}\right\} & =\operatorname{Pr}\left\{\mathbf{X}=\mathbf{x} \mid \mathbf{Y}=\mathbf{y}^{*}, p^{*}, \mathbf{K}^{*}\right\} \\
& =\frac{\operatorname{Pr}\left\{\mathbf{Y}=\mathbf{y}^{*} \mid \mathbf{X}=\mathbf{x}, p^{*}\right\} \operatorname{Pr}\left\{\mathbf{X}=\mathbf{x} \mid \mathbf{K}^{*}\right\}}{\sum_{\mathbf{x} \in \mathbf{Q}^{M N}} \operatorname{Pr}\left\{\mathbf{Y}=\mathbf{y}^{*} \mid \mathbf{X}=\mathbf{x}, p^{*}\right\} \operatorname{Pr}\left\{\mathbf{X}=\mathbf{x} \mid \mathbf{K}^{*}\right\}} .
\end{aligned}
$$

By substituting Eqs. (1) and (3) into Eq. (5), the a posteriori probability distribution is given by

$$
\begin{aligned}
\operatorname{Pr}\left\{\mathbf{X}=\mathbf{x} \mid \mathbf{Y}=\mathbf{y}^{*}\right\}=\operatorname{Pr}\left\{\mathbf{X}=\mathbf{x} \mid \mathbf{Y}=\mathbf{y}^{*}, p^{*}, \mathbf{K}^{*}\right\} \\
=\frac{\exp \left(-\frac{1}{T\left(p^{*}\right)} d\left(\mathbf{x}, \mathbf{y}^{*}\right)-K_{k}^{*} \Sigma_{n=k}^{q-1} \sigma_{n}(\mathbf{x})-\Sigma_{n=1}^{k-1} K_{n}^{*} \sigma_{n}(\mathbf{x})\right)}{\sum_{\mathbf{x} \in \mathbf{Q}^{M N}} \exp \left(-\frac{1}{T\left(p^{*}\right)} d\left(\mathbf{x}, \mathbf{y}^{*}\right)-K_{k}^{*} \Sigma_{n=k}^{q-1} \sigma_{n}(\mathbf{x})-\sum_{n=1}^{k-1} K_{n}^{*} \sigma_{n}(\mathbf{x})\right)} .
\end{aligned}
$$

In the maximum a posteriori (MAP) estimation, the restored image $\mathbf{z}^{*} \equiv\left\{z_{i, j}^{*} \mid(i, j) \in \mathbf{I}\right\}$ is estimated as follows:

$$
\mathbf{z}^{*}=\arg \max _{\mathbf{x} \in \mathbf{Q}^{M N}} \operatorname{Pr}\left\{\mathbf{X}=\mathbf{x} \mid \mathbf{Y}=\mathbf{y}^{*}, p^{*}, \mathbf{K}^{*}\right\}
$$

By introducing a set of new hyperparameters

$$
\mathbf{J}^{*} \equiv\left\{J_{n}^{*} \mid J_{n}^{*} \equiv T\left(p^{*}\right) K_{n}^{*}, n=1,2, \cdots, k\right\}
$$

instead of the hyperparameters $\mathbf{K}^{*}$, we can rewrite the a posteriori probability distribution given by Eq. (6) as follows:

$$
\operatorname{Pr}\left\{\mathbf{X}=\mathbf{x} \mid \mathbf{Y}=\mathbf{y}^{*}, p^{*}, \mathbf{K}^{*}\right\}=\frac{\exp \left(-\frac{1}{T\left(p^{*}\right)} H\left(\mathbf{x} \mid \mathbf{y}^{*}, \mathbf{J}^{*}\right)\right)}{\sum_{\mathbf{x} \in \mathbf{Q}^{M N}} \exp \left(-\frac{1}{T\left(p^{*}\right)} H\left(\mathbf{x} \mid \mathbf{y}^{*}, \mathbf{J}^{*}\right)\right)},
$$

where

$$
H\left(\mathbf{x} \mid \mathbf{y}^{*}, \mathbf{J}^{*}\right) \equiv \sum_{(i, j) \in \mathbf{I}}\left(-\delta\left(x_{\mathrm{i}, j}, y_{i, j}^{*}\right)+\phi\left(x_{i, j}, x_{i+1, j} \mid \mathbf{J}^{*}\right)+\phi\left(x_{i, j}, x_{i, j+1} \mid \mathbf{J}^{*}\right)\right)
$$




$$
\phi\left(m, n \mid \mathbf{J}^{*}\right) \equiv \sum_{l=1}^{k-1} J_{i}^{*} \delta(|m-n|, l)+J_{k}^{*} \sum_{l=k}^{q-1} \delta(|m-n|, l) .
$$

The probabilistic model described by the probability distribution (9) with Eq. (10) can be regarded as one of MRF models. It is obvious that the optimal image $\mathbf{z}^{*}$ determined by Eq. (7) depends on only the hyperparameters $\mathbf{J}^{*}$. Hence the search of the optimal image $\mathbf{z}^{*}$ can be reduced to the search of the optimal image which minimizes the energy function $H\left(\mathbf{x} \mid \mathbf{y}^{*}, \mathbf{J}^{*}\right)$ as follows:

$$
\mathbf{z}^{*}=\arg \min _{\mathbf{x} \in \mathbf{Q}^{M N}} H\left(\mathbf{x} \mid \mathbf{y}^{*}, \mathbf{J}^{*}\right) \text {. }
$$

Here the notation arg $\min _{x} f(x)$ means a minimizer $x$ of a function $f(x)$. It is equivalent to the maximum probability problem given for any positive value of $T$ as follows:

$$
\mathbf{z}^{*}=\arg \max _{\mathbf{x} \in \mathbf{Q}^{M N}} \rho\left(\mathbf{x} \mid \mathbf{y}^{*}, T, \mathbf{J}^{*}\right),
$$

where

$$
\rho\left(\mathbf{x} \mid \mathbf{y}^{*}, T, \mathbf{J}^{*}\right) \equiv \frac{\exp \left(-\frac{1}{T} H\left(\mathbf{x} \mid \mathbf{y}^{*}, \mathbf{J}^{*}\right)\right)}{\sum_{\mathbf{x} \in \mathbf{Q}^{M N}} \exp \left(-\frac{1}{T} H\left(\mathbf{x} \mid \mathbf{y}^{*}, \mathbf{J}^{*}\right)\right)} .
$$

Here the notation $\arg \max _{x} f(x)$ means a maximizer $x$ of the function $f(x)$. We should remark that the temperature $T$ in Eq. (14) is just a tool for the annealing procedure and plays a different role from $T\left(p^{*}\right)$. The restored image $\mathbf{z}^{*}$ is a function of the degraded image $\mathbf{y}^{*}$ and the hyperparameters $\mathbf{J}^{*}$.

In the maximum likelihood estimation, the hyperparameters $\mathbf{K}^{*}$ are determined from the a priori probability distribution $\operatorname{Pr}\left\{\mathbf{X}=\mathbf{x} \mid \mathbf{K}^{*}\right\}$ for the original image $\mathbf{x}$. We can regard the a priori probability distribution $\operatorname{Pr}\{\mathbf{X}$ $\left.=\mathbf{x}^{*} \mid \mathbf{K}\right\}$ as a likelihood function of hyperparameters $\mathbf{K}$ when the true original image is $\mathbf{x}^{*}$. From the standpoint of the maximum likelihood estimation, the hyperparameters $\mathbf{K}^{*}$ can be determined by

$$
\mathbf{K}^{*}=\arg \max _{\mathbf{K}} \operatorname{Pr}\left\{\mathbf{X}=\mathbf{x}^{*} \mid \mathbf{K}\right\} .
$$

From stationary conditions of $\operatorname{Pr}\left\{\mathbf{X}=\mathbf{x}^{*} \mid \mathbf{K}\right\}$ at $\mathbf{K}=\mathbf{K}^{*}$, the deterministic equations for hyperparameters $\mathbf{K}^{*}$ are given by the following equations:

$$
\begin{aligned}
& \sum_{\mathbf{x} \in \mathbf{Q}^{M N}} \sigma_{n}(\mathbf{x}) \operatorname{Pr}\left\{\mathbf{X}=\mathbf{x} \mid \mathbf{K}^{*}\right\}=\sigma_{n}\left(\mathbf{x}^{*}\right) \quad(n=1,2, \cdots, k-1), \\
& \sum_{\mathbf{x} \in \mathbf{Q}^{M N}}\left(\sum_{n=k}^{q-1} \sigma_{n}(\mathbf{x})\right) \operatorname{Pr}\left\{\mathbf{X}=\mathbf{x} \mid \mathbf{K}^{*}\right\}=\sum_{n=k}^{q-1} \sigma_{n}\left(\mathbf{x}^{*}\right) .
\end{aligned}
$$

From the values of $\sigma_{n}\left(\mathbf{x}^{*}\right)(n=1,2, \cdots, k-1)$ and $\sum_{n=\mathrm{k}}^{q-1} \sigma_{n}\left(\mathbf{x}^{*}\right)$, the hyperparameters $\mathbf{K}^{*}$ are determined by Eqs. (16) and (17). It is difficult to calculate exactly the left-hand sides of Eqs. (16) and (17). Some of the statistical-mechanical techniques for calculating them approximately are given in the next section.

For the a priori probability distribution, we have the following inequality:

$$
\begin{aligned}
& \sum_{\mathbf{x} \in \overline{\mathbf{Q}}^{M N}} \operatorname{Pr}\left\{\mathbf{X}=\mathbf{x} \mid \mathbf{K}^{*}\right\} \ln (\operatorname{Pr}\{\mathbf{X}=\mathbf{x} \mid \mathbf{K}\}) \\
& \quad \leq \sum_{\mathbf{x} \in \mathbf{Q}^{M N}} \operatorname{Pr}\left\{\mathbf{X}=\mathbf{x} \mid \mathbf{K}^{*}\right\} \ln \left(\operatorname{Pr}\left\{\mathbf{X}=\mathbf{x} \mid \mathbf{K}^{*}\right\}\right) .
\end{aligned}
$$

This inequality means that the values of hyperparameters $\mathbf{K}^{*}$, at which the a priori probability distribution $\operatorname{Pr}\{\mathbf{X}$ $\left.=\mathbf{x} \mid \mathbf{K}^{*}\right\}$ produces the original image $\mathbf{x}^{*}$, give the maximum of likelihood function $\operatorname{Pr}\left\{\mathbf{X}=\mathbf{x}^{*} \mid \mathbf{K}\right\}$. On the other hand, Nishimori and Wong [7] proved that the following inequality holds for the case of $\mathbf{Q}=\{0,1\}$

$$
M_{i, j}(p, \mathbf{K}) \leq M_{i, j}\left(p^{*}, \mathbf{K}^{*}\right),
$$

where $M_{i, j}(p, \mathbf{K})$ is a statistical overlap between the original image and the degraded image defined by

$$
\begin{aligned}
M_{i, j}(p, \mathbf{K}) \equiv & \sum_{\mathbf{x} \in \mathbf{Q}^{M N}} \sum_{\mathbf{y} \in \mathbf{Q}^{M N}} \operatorname{Pr}\left\{\mathbf{X}=\mathbf{x} \mid \mathbf{K}^{*}\right\} \operatorname{Pr}\left\{\mathbf{Y}=\mathbf{y} \mid \mathbf{X}=\mathbf{x}, p^{*}\right\} \\
& \times \delta\left(x_{i, j}, \arg \max _{n \in \mathbf{Q}}\left(\operatorname{Pr}\left\{X_{i, j}=x_{i, j} \mid \mathbf{Y}=\mathbf{y}^{*}, p, \mathbf{K}\right\}\right)\right) .
\end{aligned}
$$

Here $\operatorname{Pr}\left\{X_{i, j}=x_{i, j} \mid \mathbf{Y}=\mathbf{y}^{*}, p, \mathbf{K}\right\}$ is a posterior marginal probability distribution defined by

$$
\operatorname{Pr}\left\{X_{i, j}=x_{i, j} \mid \mathbf{Y}=\mathbf{y}^{*}, p, \mathbf{K}\right\} \equiv \sum_{\mathbf{x} \in \mathbf{Q}^{M N}} \delta\left(x_{i, j}, n\right) \operatorname{Pr}\left\{\mathbf{X}=\mathbf{x} \mid \mathbf{Y}=\mathbf{y}^{*}, p, \mathbf{K}\right\}
$$


Hereafter, the inequality (19) is referred to Nishimori-Wong's inequality. The inequality means that the restored image $\mathbf{z}^{*}$ which is closest to the original image $\mathbf{z}^{*}$ is given by the posterior marginal probability distribution $\operatorname{Pr}\left\{X_{i, j}=x_{i, j} \mid \mathbf{Y}=\mathbf{y}^{*}, p, \mathbf{K}\right\}$ for the values of hyperparameters $\mathbf{K}^{*}$, at which the a priori probability distribution $\operatorname{Pr}\left\{\mathbf{X}=\mathbf{x} \mid \mathbf{K}^{*}\right\}$ produces the original image $\mathbf{x}^{*}$, and the values of hyperparameters $p^{*}$, at which the degradation process $\operatorname{Pr}\left\{\mathbf{Y}=\mathbf{y} \mid \mathbf{X}=\mathbf{x}^{*}, p^{*}\right\}$ produces the degraded image $\mathbf{y}^{*}$ from the original image $\mathbf{x}^{*}$. The method by which the restored image $\mathbf{z}^{*}$ is determined so as to maximize the posteriori marginal probability distribution $\operatorname{Pr}\left\{X_{i, j}=x_{i, j} \mid \mathbf{Y}=\mathbf{y}^{*}, p, \mathbf{K}\right\}$ for each pixel is called the MPM estimation. From the inequalities (18) and (19), we see that the MPM estimation for the hyperparameters $\mathbf{K}^{*}$ gives the restored image $\mathbf{z}^{*}$ which is closest to the original image $\mathbf{x}^{*}$.

We give a statistical-mechanical interpretation to the maximum likelihood approach. First, although we do not assume the explicit form of the a priori probability distribution $\operatorname{Pr}\left\{\mathbf{X}=\mathbf{x} \mid \mathbf{K}^{*}\right\}$, we assume that the probability distribution satisfies the constraint conditions (16) and (17). Under these conditions, we consider the variational principle for the maximization of the entropy:

$$
-\sum_{\mathbf{x} \in \mathbf{Q} M} \operatorname{Pr}\left\{\mathbf{X}=\mathbf{x} \mid \mathbf{K}^{*}\right\} \ln \operatorname{Pr}\left\{\mathbf{X}=\mathbf{x} \mid \mathbf{K}^{*}\right\} .
$$

By introducing Lagrange multipliers $\mathbf{K}^{*}$ to ensure the constraint conditions, we obtain the explicit form of $\operatorname{Pr}\left\{\mathbf{X}=\mathbf{x} \mid \mathbf{K}^{*}\right\}$ as shown in Eq. (3). This argument is one of interpretations for the maximum likelihood approach when the a priori probability distribution has a form of Gibbs distribution in the standpoint of statistical mechanics.

\section{Probabilistic Computational Algorithms}

In this section, we will give some probabilistic computational algorithms for the optimization in Eq. (13) and a way of calculation of the left-hand sides of Eqs. (16) and (17) by means of the mean-field and the pair approximation.

It is known that annealing algorithms are able to avoid local minima. In order to adopt the MFA algorithm for the search of configuration $\mathbf{z}\left(\mathbf{y}^{*}, \mathbf{J}^{*}\right)$ minimizing the energy function $H\left(\mathbf{x} \mid \mathbf{y}^{*}, \mathbf{J}^{*}\right)$ when the degraded image $\mathbf{y}^{*}$ and the values of hyperparameters $\mathbf{J}^{*}$ are given, we introduce a one-body marginal probability distribution:

$$
\rho_{i, j}\left(n \mid \mathbf{y}^{*}, \mathbf{J}^{*}\right) \equiv \sum_{\mathbf{x} \in \mathbf{Q}} \rho\left(\mathbf{x} \mid \mathbf{y}^{*}, T, \mathbf{J}^{*}\right) \delta\left(x_{i, j}, n\right) \quad(n \in \mathbf{Q},(i, j) \in \mathbf{I}) .
$$

In the mean-field approximation, the probability distribution $\rho\left(\mathbf{x} \mid \mathbf{y}^{*}, T, \mathbf{J}^{*}\right)$ is approximately expressed as

$$
\rho\left(\mathbf{x} \mid \mathbf{y}^{*}, T, \mathbf{J}^{*}\right) \simeq \prod_{(i, j) \in \mathbf{I}} \rho_{i, j}\left(x_{i, j} \mid \mathbf{y}^{*}, T, \mathbf{J}^{*}\right) .
$$

The Gibbs distribution $\rho\left(\mathbf{x} \mid \mathbf{y}^{*}, T, \mathbf{J}^{*}\right)$ satisfies the variational principle for the minimization of the following free energy:

$$
\mathscr{F}[\rho] \equiv \sum_{\mathbf{x} \in \mathbf{Q}, N=} \rho\left(\mathbf{x} \mid \mathbf{y}^{*}, \mathbf{J}^{*}\right)\left\{H\left(\mathbf{x} \mid \mathbf{y}^{*}, T, \mathbf{J}^{*}\right)+T \ln \rho\left(\mathbf{x} \mid \mathbf{y}^{*}, T, \mathbf{J}^{*}\right)\right\}
$$

under the normalization condition for $\rho\left(\mathbf{x} \mid \mathbf{y}^{*}, T, \mathbf{J}^{*}\right)$. By applying Eqs. (23) and (24) to the right-hand side of Eq. (25), the free energy $\mathscr{F}[\rho]$ can approximately be rewritten as

$$
\begin{aligned}
\mathscr{\mathscr { F }}[\rho] & \simeq \widetilde{\mathscr{F}}_{\mathrm{MF}}\left[\left\{\rho_{i, j}\right\}\right] \\
& \equiv \sum_{(i, j) \in \mathbf{I}}\left\{-\delta\left(n, y_{i, j}^{*}\right) \rho_{i, j}\left(n \mid \mathbf{y}^{*}, T, \mathbf{J}^{*}\right)+\sum_{n \in \mathbf{Q}} \sum_{n^{\prime} \in \mathbf{Q}}\left(\sum_{m=1}^{k-1} J_{m}^{*} \delta\left(\left|n-n^{\prime}\right|, m\right)+J_{k}^{*} \sum_{m=k}^{q-1} \delta\left(\left|n-n^{\prime}\right|, m\right)\right)\right. \\
& \times \rho_{i, j}\left(n \mid \mathbf{y}^{*}, T, \mathbf{J}^{*}\right)\left(\rho_{i+1, j}\left(n^{\prime} \mid \mathbf{y}^{*}, T, \mathbf{J}^{*}\right)+\rho_{i, j+1}\left(n^{\prime} \mid \mathbf{y}^{*}, T, \mathbf{J}^{*}\right)\right) \\
& \left.+T \sum_{n \in \mathbf{Q}} \rho_{i, j}\left(n \mid \mathbf{y}^{*}, T, \mathbf{J}^{*}\right) \ln \rho_{i, j}\left(n \mid \mathbf{y}^{*}, T, \mathbf{J}^{*}\right)\right\} .
\end{aligned}
$$

By taking the first variation of the free energy $\mathscr{F}_{\mathrm{MF}}\left[\left\{\rho_{i, j}\right\}\right]$ with respect to $\rho_{i, j}(n)$ under the normalization condition for the marginal probability distribution, the deterministic mean-field equations for the set of one-body marginal distribution functions $\left\{\rho_{i, j}\left(n \mid \mathbf{y}^{*}, T, \mathbf{J}^{*}\right) \mid(i, j) \in \mathbf{I}, n \in \mathbf{Q}\right\}$ are obtained as follows:

$$
\begin{aligned}
& \rho_{i, j}\left(n \mid \mathbf{y}^{*}, T, \mathbf{J}^{*}\right)=\frac{\exp \left(-\frac{1}{T} H_{i, j}\left(n \mid \rho_{i^{\prime}, j^{\prime}}\left(m \mid \mathbf{y}^{*}, T, \mathbf{J}^{*}\right), m \in \mathbf{Q},\left(i^{\prime}, j^{\prime}\right) \in \mathbf{c}_{i, j}\right)\right)}{\sum_{n \in \mathbf{Q}} \exp \left(-\frac{1}{T} H_{i, j}\left(n \mid \rho_{i^{\prime}, j^{\prime}}\left(m \mid \mathbf{y}^{*}, T, \mathbf{J}^{*}\right), m \in \mathbf{Q},\left(i^{\prime}, j^{\prime}\right) \in \mathbf{c}_{i, j}\right)\right)}, \\
& H_{i, j}\left(n \mid \rho_{i^{\prime}, j^{\prime}}^{(1)}\left(m \mid \mathbf{y}^{*}, T, \mathbf{J}^{*}\right), m \in \mathbf{Q},\left(i^{\prime}, j^{\prime}\right) \in \mathbf{c}_{i, j}\right) \\
& =-\delta\left(n, y_{i, j}^{*}\right)+\sum_{\left(i^{\prime}, j^{\prime}, \in \mathbf{c}_{i, j}\right.} \sum_{m=1}^{k-1} J_{m}^{*}\left(\rho_{i^{\prime}, j^{\prime}}\left(n+m \mid \mathbf{y}^{*}, T, \mathbf{J}^{*}\right)+\rho_{i^{\prime}, j^{\prime}}\left(n-m \mid \mathbf{y}^{*}, T, \mathbf{J}^{*}\right)\right)
\end{aligned}
$$




$$
+J_{k}^{*} \sum_{\left(i^{\prime}, j^{\prime}\right) \in \mathbf{c}_{i, j}} \sum_{m=k}^{q-1}\left(\rho_{i^{\prime}, j^{\prime}}\left(n+m \mid \mathbf{y}^{*}, T, \mathbf{J}^{*}\right)+\rho_{i^{\prime}, j^{\prime}}\left(n-m \mid \mathbf{y}^{*}, T, \mathbf{J}^{*}\right)\right),
$$

where

$$
\mathbf{c}_{i, j}=\{(i+1, j),(i-1, j),(i, j+1),(i, j-1)\} .
$$

By solving Eqs. (27)-(29) at a sufficiently small positive value of $T$ by using the annealing procedure, we obtain an approximate optimal solution from the following equation

$$
z_{i, j}^{*}=\arg \max _{n \in \mathbf{Q}} \rho_{i, j}\left(n \mid \mathbf{y}^{*}, T, \mathbf{J}^{*}\right) .
$$

We summarize the algorithm as follows:

\section{MFA algorithm}

Step 1: Initialize so that $r \leftarrow 1, T \leftarrow 2.5 J_{k}^{*}$ and

$$
\rho_{i, j}(n) \leftarrow \frac{\exp \left(\frac{1}{T} \delta\left(n, y_{i, j}\right)\right)}{\Sigma_{\xi \in \mathbf{Q}} \exp \left(\frac{1}{T} \delta\left(n, y_{i, y}\right)\right)}(n \in \mathbf{Q},(i, j) \in \mathbf{I}) .
$$

Step 2: Update the value of $r$ to $r+1$ and set $T \leftarrow\left(2.5 J_{k}^{*} \ln 2\right) / \ln (r+1)$. For each site $(i, j) \in \mathbf{I}$, update $\rho_{i, j}(n)(n \in \mathbf{Q})$ according to the equation

$$
\rho_{i, j}(n) \leftarrow \frac{\exp \left[-\frac{1}{T} H_{i, j}\left(n \mid \rho_{i^{\prime}, j^{\prime}}(m), m \in \mathbf{Q},\left(i^{\prime}, j^{\prime}\right) \in \mathbf{I}\right)\right]}{\Sigma_{n \in \mathbf{Q}} \exp \left[-\frac{1}{T} H_{i, j}\left(n \mid \rho_{i^{\prime}, j^{\prime}}(m), m \in \mathbf{Q},\left(i^{\prime}, j^{\prime}\right) \in \mathbf{I}\right)\right]}(n \in \mathbf{Q},(i, j) \in \mathbf{I}) .
$$

Step 3: If $r=R$, then $z_{i, j}^{*} \leftarrow \arg \max _{n \in \mathbf{Q}}\left(\rho_{i, j}(n)\right)$ for $(i, j) \in \mathbf{I}$, and stop, else go to Step 2 .

Next, we explain the pair approximation. A set of two-body marginal distributions $\left\{\boldsymbol{\rho}_{i, j}^{i^{\prime}, j^{\prime}}\left(m, m^{\prime} \mid \mathbf{y}^{*}, T, \mathbf{J}^{*}\right) \mid\right.$ $\left.(i, j) \in \mathbf{I}, m \in \mathbf{Q},\left(i^{\prime}, j^{\prime}\right)=(i+1, j),(i, j+1)\right\}$ is defined by

$$
\begin{aligned}
& \rho_{i, j}^{i+1, j}\left(m, m^{\prime} \mid \mathbf{y}^{*}, T, \mathbf{J}^{*}\right) \equiv \sum_{\mathbf{x} \in \mathbf{Q}^{M N}} \rho\left(\mathbf{x} \mid \mathbf{y}^{*}, T, \mathbf{J}^{*}\right) \delta\left(x_{i, j}, m\right) \delta\left(x_{i+1, j}, m^{\prime}\right), \\
& \rho_{i, j}^{i, j+1}\left(m, m^{\prime} \mid \mathbf{y}^{*}, T, \mathbf{J}^{*}\right) \equiv \sum_{\mathbf{x} \in \mathbf{Q}^{M N}} \rho\left(\mathbf{x} \mid \mathbf{y}^{*}, T, \mathbf{J}^{*}\right) \delta\left(x_{i, j}, m\right) \delta\left(x_{i, j+1}, m^{\prime}\right) .
\end{aligned}
$$

In the pair approximation, the probability distribution $\rho\left(\mathbf{x} \mid \mathbf{y}^{*}, T, \mathbf{J}^{*}\right)$ is approximately expressed as

$$
\begin{aligned}
\rho\left(\mathbf{x} \mid \mathbf{y}^{*}, T, \mathbf{J}^{*}\right) \simeq & \prod_{(i, j) \in \mathbf{I}} \rho_{i, j}\left(x_{i, j} \mid \mathbf{y}^{*}, T, \mathbf{J}^{*}\right) \prod_{(i, j) \in \mathbf{I}} \frac{\rho_{i, j}^{i+1, j}\left(x_{i, j}, x_{i+1, j} \mid \mathbf{y}^{*}, T, \mathbf{J}^{*}\right)}{\left.\rho_{i, j} \mid \mathbf{y}^{*}, T, \mathbf{J}^{*}\right) \rho_{i+1, j}\left(x_{i+1, j} \mid \mathbf{y}^{*}, T, \mathbf{J}^{*}\right)} \\
& \times \prod_{(i, j) \in \mathbf{I}} \frac{\rho_{i, j}^{i, j+1}\left(x_{i, j}, x_{i, j+1} \mid \mathbf{y}^{*}, T, \mathbf{J}^{*}\right)}{\rho_{i, j}\left(x_{i, j} \mid \mathbf{y}^{*}, T, \mathbf{J}^{*}\right) \rho_{i, j+1}\left(x_{i, j+1} \mid \mathbf{y}^{*}, T, \mathbf{J}^{*}\right)} .
\end{aligned}
$$

The marginal probability distributions $\rho_{i, j}\left(x_{i, j} \mid \mathbf{y}^{*}, T, \mathbf{J}^{*}\right)$ and $\rho_{i, j}^{i^{\prime}, j^{\prime}}\left(x_{i, j}, x_{i^{\prime}, j^{\prime}} \mid \mathbf{y}^{*}, T, \mathbf{J}^{*}\right)$ satisfy the normalization conditions:

$$
\begin{aligned}
& \sum_{m \in \mathbf{Q}} \rho_{i, j}\left(m \mid \mathbf{y}^{*}, T, \mathbf{J}^{*}\right)=1, \\
& \sum_{m \in \mathbf{Q}} \sum_{m^{\prime} \in \mathbf{Q}} \rho_{i, j^{\prime}}^{i^{\prime}, j^{\prime}}\left(m, m \mid \mathbf{y}^{*}, T, \mathbf{J}^{*}\right)=1\left(\left(i^{\prime}, j^{\prime}\right)=(i+1, j),(i, j+1)\right),
\end{aligned}
$$

and the reducibility conditions:

$$
\begin{aligned}
\rho_{i, j}^{(1)}\left(m \mid \mathbf{y}^{*}, T, \mathbf{J}^{*}\right) & =\sum_{m^{\prime} \in \mathbf{Q}} \rho_{i, j}^{i+1, j}\left(m, m^{\prime} \mid \mathbf{y}^{*}, T, \mathbf{J}^{*}\right)=\sum_{m^{\prime} \in \mathbf{Q}} \rho_{i-1, j}^{i, j}\left(m^{\prime}, m \mid \mathbf{y}^{*}, T, \mathbf{J}^{*}\right) \\
& =\sum_{m^{\prime} \in \mathbf{Q}} \rho_{i, j}^{i, j+1}\left(m, m^{\prime} \mid \mathbf{y}^{*}, T, \mathbf{J}^{*}\right)=\sum_{m^{\prime} \in \mathbf{Q}} \rho_{i, j-1}^{i, j}\left(m^{\prime}, m \mid \mathbf{y}^{*}, T, \mathbf{J}^{*}\right) \quad(m \in \mathbf{Q}) .
\end{aligned}
$$

By applying Eqs. (23), (32), (33) and (34) to the right-hand side of Eq. (25), the free energy $\mathscr{F}[\rho]$ can approximately be rewritten as

$$
\begin{aligned}
\mathscr{F}[\rho] & \simeq \mathscr{F}_{\mathrm{P}}\left[\left\{\rho_{i, j}\right\}\right] \\
& \equiv \sum_{(i, j) \in \mathbf{I}}\left(-\delta\left(n, y_{i, j}^{*}\right) \rho_{i, j}(n)\right.
\end{aligned}
$$




$$
\begin{aligned}
& +\sum_{n \in \mathbf{Q}} \sum_{n^{\prime} \in \mathbf{Q}}\left(\sum_{m=1}^{k-1} J_{m}^{*} \delta\left(\left|n-n^{\prime}\right|, m\right)+J_{k}^{*} \sum_{m=k}^{q-1} \delta\left(\left|n-n^{\prime}\right|, m\right)\right) \\
& \times\left(\rho_{i, j}^{i+1, j}\left(n, n^{\prime} \mid \mathbf{y}^{*}, T, \mathbf{J}^{*}\right)+\rho_{i, j}^{i, j+1}\left(n, n^{\prime} \mid \mathbf{y}^{*}, T, \mathbf{J}^{*}\right)\right) \\
& +T \sum_{n \in \mathbf{Q}} \rho_{i, j}\left(n \mid \mathbf{y}^{*}, T, \mathbf{J}^{*}\right) \ln \rho_{i, j}\left(n \mid \mathbf{y}^{*}, T, \mathbf{J}^{*}\right) \\
& +T\left(\sum_{n \in \mathbf{Q}} \sum_{n^{\prime} \in \mathbf{Q}} \rho_{i, j}^{i+1, j}\left(n, n^{\prime} \mid \mathbf{y}^{*}, T, \mathbf{J}^{*}\right) \ln \rho_{i, j}^{i+1, j}\left(n, n^{\prime} \mid \mathbf{y}^{*}, T, \mathbf{J}^{*}\right)\right. \\
& -\sum_{n \in \mathbf{Q}} \rho_{i, j}\left(n^{\prime} \mid \mathbf{y}^{*}, T, \mathbf{J}^{*}\right) \ln \rho_{i, j}\left(n \mid \mathbf{y}^{*}, T, \mathbf{J}^{*}\right) \\
& \left.-\sum_{n \in \mathbf{Q}} \rho_{i+1, j}\left(n \mid \mathbf{y}^{*}, T, \mathbf{J}^{*}\right) \ln \rho_{i+1, j}\left(n \mid \mathbf{y}^{*}, T, \mathbf{J}^{*}\right)\right) \\
& +T\left(\sum_{n \in \mathbf{Q}} \sum_{n^{\prime} \in \mathbf{Q}} \rho_{i, j}^{i, j+1}\left(n, n^{\prime} \mid \mathbf{y}^{*}, T, \mathbf{J}^{*}\right) \ln \rho_{i, j}^{i, j+1}\left(n, n^{\prime} \mid \mathbf{y}^{*}, T, \mathbf{J}^{*}\right)\right. \\
& -\sum_{n \in \mathbf{Q}} \rho_{i, j}\left(n \mid \mathbf{y}^{*}, T, \mathbf{J}^{*}\right) \ln \rho_{i, j}\left(n \mid \mathbf{y}^{*}, T, \mathbf{J}^{*}\right) \\
& \left.\left.-\sum_{n \in \mathbf{Q}} \rho_{i, j+1}\left(n \mid \mathbf{y}^{*}, T, \mathbf{J}^{*}\right) \ln \rho_{i, j+1}\left(n \mid \mathbf{y}^{*}, T, \mathbf{J}^{*}\right)\right)\right\} .
\end{aligned}
$$

By taking the first variation of $\mathscr{F}_{\mathrm{P}}\left[\left\{\rho_{i, j}, \rho_{i, j}^{i, j+1}, \rho_{i, j}^{i+1, j}\right\}\right]$ with respect to $\rho_{i, j}\left(x_{i, j} \mid \mathbf{y}^{*}, T, \mathbf{J}^{*}\right), \rho_{i, j}^{i+1, j}\left(x_{i, j}, x_{i+1, j} \mid \mathbf{y}^{*}, T\right.$, $\left.\mathbf{J}^{*}\right)$ and $\rho_{i, j}^{i, j+1}\left(x_{i, j}, x_{i, j+1} \mid \mathbf{y}^{*}, T, \mathbf{J}^{*}\right)$ under the normalization conditions (35) and (36) and reducibility conditions (37), the one-body marginal distribution functions $\rho_{i, j}\left(m \mid \mathbf{y}^{*}, T, \mathbf{J}^{*}\right)$ and the two-body marginal distribution functions $\rho_{i, j}^{i^{\prime}, j^{\prime}}\left(m, m^{\prime} \mid \mathbf{y}^{*}, T, \mathbf{J}^{*}\right)\left(\left(i^{\prime}, j^{\prime}\right) \in\{(i+1, j),(i, j+1)\}\right)$ in the system with the energy function $H\left(\mathbf{x} \mid \mathbf{y}^{*}, \mathbf{J}^{*}\right)$ given by Eq. (10), at temperature $T$, are expressed as follows [15, 16]:

$$
\begin{aligned}
\rho_{i, j}\left(m \mid \mathbf{y}^{*}, T, \mathbf{J}^{*}\right)=\frac{\exp \left(-\frac{1}{T} H_{i, j}(m)\right)}{\sum_{m \in \mathbf{Q}} \exp \left(-\frac{1}{T} H_{i, j}(m)\right)}(m \in \mathbf{Q}), \\
\rho_{i, j^{\prime}}^{i^{\prime} j^{\prime}}\left(m, m^{\prime} \mid \mathbf{y}^{*}, T, \mathbf{J}^{*}\right)=\frac{\exp \left(-\frac{1}{T} H_{i, j}^{i^{i}, j^{\prime}}\left(m, m^{\prime}\right)\right)}{\sum_{m \in \mathbf{Q}} \Sigma_{m^{\prime} \in \mathbf{Q}} \exp \left(-\frac{1}{T} H_{i, j}^{i^{\prime}, j^{\prime}}\left(m, m^{\prime}\right)\right)} \\
\left(m, m^{\prime} \in \mathbf{Q},\left(i^{\prime}, j^{\prime}\right)=(i+1, j),(i, j+1)\right),
\end{aligned}
$$

where

$$
\begin{aligned}
& H_{i, j}(m) \equiv-\delta\left(m, y_{i, j}^{*}\right)-\sum_{\left(i^{\prime}, j^{\prime}\right) \in \mathbf{c}^{i, j}} \lambda_{i^{\prime}, j^{\prime}}^{i, j}(m), \\
& H_{i, j}^{i+1, j}\left(m, m^{\prime}\right) \equiv-\delta\left(m, y_{i, j}^{*}\right)-\delta\left(m^{\prime}, y_{i+1, j}^{*}\right)-\phi\left(m, m^{\prime} \mid \mathbf{J}^{*}\right) \\
&-\sum_{\left(i^{\prime}, j^{\prime}\right) \in \mathbf{c}_{i, j}(i+1, j)} \lambda_{i^{\prime}, j^{\prime}}^{i, j}(m)-\sum_{\left(i^{\prime}, j^{\prime}\right) \in \mathbf{c}_{i+1, j} \backslash(i, j)} \lambda_{i^{\prime}, j^{\prime}, j}^{i+1, j}\left(m^{\prime}\right), \\
& H_{i, j}^{i, j+1}\left(m, m^{\prime}\right) \equiv-\delta\left(m, y_{i, j}^{*}\right)-\delta\left(m^{\prime}, y_{i, j+1}^{*}\right)-\phi\left(m, m^{\prime} \mid \mathbf{J}^{*}\right) \\
&-\sum_{\left(i^{\prime}, j^{\prime}\right) \in \mathbf{c}_{i_{j}, j}(i, j+1)} \lambda_{i^{\prime}, j^{\prime}}^{i, j}(m)-\sum_{\left(i^{\prime}, j^{\prime}\right) \in \mathbf{c}_{i, j+1} \backslash(i, j)} \lambda_{i^{\prime}, j^{\prime}}^{i, j+1}\left(m^{\prime}\right) .
\end{aligned}
$$

The set $\left\{\lambda_{i^{\prime}, j^{\prime}}^{i, j}(m) \mid(i, j) \in \mathbf{I},\left(i^{\prime}, j^{\prime}\right) \in \mathbf{c}_{i, j}, m \in \mathbf{Q}\right\}$ is determined so as to satisfy the reducibility conditions (37). For a sufficiently small positive $T$, we can obtain the approximate optimal image $\mathbf{z}(J)$ by using Eq. (30).

In order to reduce Eqs. (37)-(43) to recursion formula, we introduce the orthonormal polynomials $\Phi_{m}(n)$ given in [17] as

$$
\begin{aligned}
\Phi_{m}(n) \equiv & \sqrt{\frac{(2 m+1)[(q-1) !]^{2}}{(q+m) !(q-m-1) !}} \\
& \times \sum_{l=0}^{m}(-1)^{l+m}\left(\begin{array}{c}
m \\
l
\end{array}\right)\left(\begin{array}{c}
m+l \\
l
\end{array}\right) \frac{n !(q-l-1) !}{\Gamma(n-l+1)(q-1) !}
\end{aligned}
$$

for $n \in \mathbf{Q}$ and also by the recursion formula: 


$$
\Phi_{m}(n) \equiv \frac{\Psi_{m}(n)}{\sqrt{\sum_{n \in \mathbf{Q}} \Psi_{m}(n)^{2}}} .
$$

Here we have the recursion formula as follows:

$$
\begin{aligned}
(m+1)(q-1-m) \Psi_{m+1}(n)= & -(2 n-q+1)(2 m+1) \Psi_{m}(n) \\
& -m(q+m) \Psi_{m-1}(n) \quad(n \in \mathbf{Q}),
\end{aligned}
$$

where

$$
\Psi_{0}(n)=1, \quad \Psi_{1}(n)=1-\frac{2}{q-1} n .
$$

From the variational principle, the one-body distribution functions $\rho_{i, j}(m)$ and the two-body distribution functions, $\rho_{i, j}^{i+1, j}\left(m, m^{\prime}\right)$ and $\rho_{i, j}^{i, j+1}\left(m, m^{\prime}\right)$, are expressed as

$$
\begin{aligned}
& H_{i, j}(m)=-\delta\left(m, y_{i, j}^{*}\right)-\sum_{\left(i^{\prime}, j^{\prime}\right) \in \mathbf{c}_{i, j}} \sum_{n \in \mathbf{Q}} \tilde{\lambda}_{i^{\prime}, j^{\prime}}^{i, j}(n) \Phi_{n}(m), \\
& H_{i, j}^{i+1, j}\left(m, m^{\prime}\right)=-\delta\left(m, y_{i, j}^{*}\right)-\delta\left(m^{\prime}, y_{i+1, j}^{*}\right)-\phi\left(m, m^{\prime} \mid \mathbf{J}^{*}\right) \\
& -\sum_{\left(i^{\prime}, j^{\prime}\right) \in \mathbf{c}_{i, j}(i+1, j)} \sum_{n \in \mathbf{Q}} \tilde{\lambda}_{i^{\prime}, j^{\prime}}^{i, j}(n) \Phi_{n}(m)-\sum_{\left(i^{\prime}, j^{\prime}\right) \in \mathbf{c}_{i+1, j} \backslash(i, j)} \sum_{n^{\prime} \in \mathbf{Q}} \tilde{\lambda}_{i^{\prime}, j^{\prime}}^{i+1, j}\left(n^{\prime}\right) \Phi_{n}\left(m^{\prime}\right) \\
& H_{i, j}^{i, j+1}\left(m, m^{\prime}\right)=-\delta\left(m, y_{i, j}^{*}\right)-\delta\left(m^{\prime}, y_{i, j+1}^{*}\right)-\phi\left(m, m^{\prime} \mid \mathbf{J}^{*}\right) \\
& -\sum_{\left(i^{\prime}, j^{\prime}\right) \in \mathbf{c}_{i, j} \backslash(i, j+1)} \sum_{n \in \mathbf{Q}} \tilde{\lambda}_{i^{\prime}, j^{\prime}}^{i, j}(n) \Phi_{n}(m)-\sum_{\left(i^{\prime}, j^{\prime}\right) \in \mathbf{c}_{i, j+1} \backslash(i, j)} \sum_{n^{\prime} \in \mathbf{Q}} \tilde{\lambda}_{i^{\prime}, j^{\prime}}^{i, j}\left(n^{\prime}\right) \Phi_{n}\left(m^{\prime}\right), \\
& \tilde{\lambda}_{i+1, j}^{i, j}(m)=-\sum_{\left\{\left(i^{\prime}, j^{\prime}\right) \mid\left(i^{\prime}, j^{\prime}\right) \in \mathbf{c}_{i+1, j} \backslash(i, j)\right\}} \tilde{\lambda}_{i, j^{\prime}}^{i^{\prime} j^{\prime}}(m) \\
& -\sum_{n=0}^{q-1} \Phi_{m}(n) \ln \left(\sum_{n^{\prime}=0}^{q-1} \Phi_{n^{\prime}}(n) \sum_{l=0}^{q-1} \sum_{l^{\prime}=0}^{q-1} \Phi_{n^{\prime}}\left(l^{\prime}\right) \rho_{i, j}^{i+1, j}\left(l, l^{\prime} \mid \mathbf{y}^{*}, T, \mathbf{J}^{*}\right)\right), \\
& \tilde{\lambda}_{i, j}^{i+1, j}(m)=-\sum_{\left\{\left(i^{\prime}, j^{\prime}\right) \mid\left(i^{\prime}, j^{\prime}\right) \in \mathbf{c}_{i, j}(i+1, j)\right\}} \tilde{\lambda}_{i, j^{\prime}}^{i^{\prime}, j^{\prime}}(m) \\
& -\sum_{n=0}^{q-1} \Phi_{m}(n) \ln \left(\sum_{n^{\prime}=0}^{q-1} \Phi_{n^{\prime}}(n) \sum_{l=0}^{q-1} \sum_{l^{\prime}=0}^{q-1} \Phi_{n^{\prime}}(l) \rho_{i, j}^{i+1, j}\left(l, l^{\prime} \mid \mathbf{y}^{*}, T, \mathbf{J}^{*}\right)\right) \text {, } \\
& \tilde{\lambda}_{i, j+1}^{i, j}(m)=-\sum_{\left\{\left(i^{\prime}, j^{\prime}\right) \mid\left(i^{\prime}, j^{\prime}\right) \in \mathbf{c}_{i, j+1} \backslash(i, j)\right\}} \tilde{\lambda}_{i, j}^{i^{\prime}, j^{\prime}}(m) \\
& -\sum_{n=0}^{q-1} \Phi_{m}(n) \ln \left(\sum_{n^{\prime}=0}^{q-1} \Phi_{n^{\prime}}(n) \sum_{l=0}^{q-1} \sum_{l^{\prime}=0}^{q-1} \Phi_{n^{\prime}}\left(l^{\prime}\right) \rho_{i, j}^{i, j+1}\left(l, l^{\prime} \mid \mathbf{y}^{*}, T, \mathbf{J}^{*}\right)\right), \\
& \tilde{\lambda}_{i, j}^{i, j+1}(m)=-\sum_{\left\{\left(i^{\prime}, j^{\prime}\right) \mid\left(i^{\prime}, j^{\prime}\right) \in \mathbf{e}_{i, j} \backslash(i, j+1)\right\}} \tilde{\lambda}_{i, j}^{i^{\prime}, j^{\prime}}(m) \\
& -\sum_{n=0}^{q-1} \Phi_{m}(n) \ln \left(\sum_{n^{\prime}=0}^{q-1} \Phi_{n^{\prime}}(n) \sum_{i=0}^{q-1} \sum_{l^{\prime}=0}^{q-1} \Phi_{n^{\prime}}(l) \rho_{i, j}^{i, j+1}\left(l, l^{\prime} \mid \mathbf{y}^{*}, T, \mathbf{J}^{*}\right)\right) .
\end{aligned}
$$

We use the pair approximation to calculate the quantities the left-hand sides of Eqs. (16) and (17). The twobody marginal probability distributions of the a priori probability defined by

$$
\begin{aligned}
& \rho_{i, j}^{i+1, j}\left(m, m^{\prime} \mid \mathbf{K}^{*}\right) \equiv \sum_{\mathbf{x} \in \mathbf{Q}_{M N}} \operatorname{Pr}\left\{\mathbf{X}=\mathbf{x} \mid \mathbf{K}^{*}\right\} \delta\left(x_{i, j}, m\right) \delta\left(x_{i+1, j}, m^{\prime}\right), \\
& \rho_{i, j}^{i, j+1}\left(m, m^{\prime} \mid \mathbf{K}^{*}\right) \equiv \sum_{\mathbf{x} \in \mathbf{Q}^{M N}} \operatorname{Pr}\left\{\mathbf{X}=\mathbf{x} \mid \mathbf{K}^{*}\right\} \delta\left(x_{i, j}, m\right) \delta\left(x_{i, j+1}, m^{\prime}\right),
\end{aligned}
$$

should be independent of $(i, j)$, because the system, which is given by the a priori probability distribution $\operatorname{Pr}\{\mathbf{X}$ $\left.=\mathbf{x} \mid \mathbf{K}^{*}\right\}$, has no external field. Hence we set $\rho_{i, j}^{i, j+1}\left(m, m^{\prime} \mid \mathbf{K}^{*}\right)=\rho_{i, j}^{i+1, j}\left(m, m^{\prime} \mid \mathbf{K}^{*}\right)=\rho^{(2)}\left(m, m^{\prime} \mid \mathbf{K}\right)$. By using Eqs. (32) and (33), the left-hand sides of Eqs. (16) and (17) are expressed as

$$
\begin{aligned}
& 2 M N \sum_{m \in \mathbf{Q}} \sum_{m^{\prime} \in \mathbf{Q}} \sum_{n=k}^{q-1} \delta\left(\left|m-m^{\prime}\right|, n\right) \rho^{(2)}\left(m, m^{\prime} \mid \mathbf{K}^{*}\right)=\sum_{n=k}^{q-1} \sigma_{n}\left(\mathbf{x}^{*}\right), \\
& 2 M N \sum_{m \in \mathbf{Q}} \sum_{m^{\prime} \in \mathbf{Q}} \delta\left(\left|m-m^{\prime}\right|, n\right) \rho^{(2)}\left(m, m^{\prime} \mid \mathbf{K}^{*}\right)=\sigma_{n}\left(\mathbf{x}^{*}\right) \quad(n=1,2, \cdots, k-1) .
\end{aligned}
$$

A set of deterministic equations for the two-body marginal probability distributions $\rho^{(2)}\left(m, m^{\prime} \mid \mathbf{K}\right)$ is given as follows:

$$
\rho^{(2)}\left(m, m^{\prime} \mid \mathbf{K}^{*}\right)=\frac{\exp \left(\phi\left(m, m^{\prime} \mid \mathbf{K}^{*}\right)+3 \lambda(m)+3 \lambda\left(m^{\prime}\right)\right)}{\sum_{n \in \mathbf{Q}} \Sigma_{n^{\prime} \in \mathbf{Q}} \exp \left(\phi\left(n, n^{\prime} \mid \mathbf{K}^{*}\right)+3 \lambda(n)+3 \lambda\left(n^{\prime}\right)\right)} \quad\left(m, m^{\prime} \in \mathbf{Q}\right),
$$




$$
\frac{\exp (4 \lambda(m))}{\sum_{n \in \mathbf{Q}} \exp (4 \lambda(n))}=\frac{\sum_{m \in \mathbf{Q}} \exp \left(-\phi\left(m, m^{\prime} \mid \mathbf{K}^{*}\right)+3 \lambda(m)+3 \lambda\left(m^{\prime}\right)\right)}{\sum_{n \in \mathbf{Q}} \Sigma_{n^{\prime} \in \mathbf{Q}} \exp \left(-\phi\left(n, n^{\prime} \mid \mathbf{K}^{*}\right)+3 \lambda(n)+3 \lambda\left(n^{\prime}\right)\right)} \quad(m \in \mathbf{Q}) .
$$

By solving the simultanenous non-linear Eqs. (55), (56), (57) and (58) numerically, we obtain the approximate maximum likelihood estimates $\mathbf{K}^{*}$.

\section{Iterated Conditional Models}

In this section, we briefly explain the interated conditional modes (ICM) as a simple deterministic algorithm for the optimization problem (12) and its extension to the cluster version.

The optimization problem (12) can be reduced to the following iterative equation:

$$
z_{i, j}^{*}=\arg \min _{x_{i, j} \in \mathbf{Q}} H_{i, j}\left(x_{i, j} \mid x_{i^{\prime}, j^{\prime}}=z_{i^{\prime}, j^{\prime}}^{*},\left(i^{\prime}, j^{\prime}\right) \in \mathbf{I} \backslash(i, j)\right) \text {. }
$$

Here, the notation $H_{i, j}\left(x_{i, j} \mid x_{i^{\prime}, j^{\prime}}=z_{i^{\prime}, j^{\prime}}^{*},\left(i^{\prime}, j^{\prime}\right) \in \mathbf{I} \backslash(i, j)\right)$ denotes a value of $H(\mathbf{x})$ when the variable $x_{i^{\prime}, j}$ is set to the value of $z_{i^{\prime}, j^{\prime}}^{*}$ for any pixel $\left(i^{\prime}, j^{\prime}\right) \in \mathbf{I} \backslash(i, j)$ and the energy function $H(\mathbf{x})$ is regarded as a function of $x_{i, j}$. From the above iterative equation, we can construct the ICM algorithm [2], which is the simplest algorithms for the optimization (12). From these deterministic equations, we obtain the following iterative algorithm which is given by the recursion formula (59) for $\mathbf{z}^{(r)}=\left\{z_{i, j}^{(r)} \mid(i, j) \in \mathbf{I}\right\}$.

\section{ICM algorithm}

Step 1: Initialize so that $\mathbf{z}^{(0)} \leftarrow \mathbf{y}$ and $r \leftarrow 0$.

Step 2: Update the value of $r$ to $r+1$. For each site $(i, j) \in \mathbf{I}$, update $z_{i, j}^{(r)}$ according to the equation

$$
z_{i, j}^{(r)} \leftarrow \arg \min _{x_{i, j} \in \mathbf{Q}} H_{i, j}\left(x_{i, j} \mid x_{i^{\prime}, j^{\prime}}=z_{i^{\prime}, j^{\prime}}^{(r)},\left(i^{\prime}, j^{\prime}\right) \in \mathbf{c}_{i, j}\right) \text {. }
$$

Step 3: Set $\mathbf{z}^{*} \leftarrow \mathbf{z}^{(r)}$ and $R \leftarrow r$, and stop if $\mathbf{z}^{(r)}=\mathbf{z}^{(r-1)}$.

Step 4: Go to Step 2.

We remark that the configuration $z_{i, j}^{(r)}$ at each site $(i, j)$ is updated sequentially in the step 2. In Ref. [18], the ICM algorithm was extended to the cluster varsion ICM algorithm, which is constructed from the following iterative equation:

$$
\mathbf{z}_{\mathbf{c}}^{*}=\arg \min _{\mathbf{x}_{\mathrm{i}} \in \mathbf{Q}^{\mathrm{c} l}} H_{\mathrm{c}}\left(\mathbf{x}_{\mathbf{c}} \mid x_{i^{\prime}, j^{\prime}}=z_{i^{\prime}, j^{\prime}}^{*},\left(i^{\prime}, j^{\prime}\right) \in \mathbf{I} \backslash \mathbf{c}\right),
$$

where $\mathbf{x}_{\mathbf{c}} \equiv\left\{x_{i, j} \mid(i, j) \in \mathbf{c}\right\}$. Here, the notation $H_{\mathbf{c}}\left(x_{\mathbf{c}} \mid x_{i^{\prime}, j^{\prime}}=z_{i^{\prime}, j^{\prime}}^{*},\left(i^{\prime}, j^{\prime}\right) \in \mathbf{I} \backslash \mathbf{c}\right)$ denotes a value of $H(\mathbf{x})$ when the variable $x_{i^{\prime}, j^{\prime}}$ is set to the value of $z_{i^{\prime}, j^{\prime}}$ for any pixel $\left(i^{\prime}, j^{\prime}\right) \in \mathbf{I} \backslash \mathbf{c}$ and the energy function $H(\mathbf{x})$ is regarded as a function of $x_{\mathrm{c}}$. In Ref. [18], the cluster version ICM algorithm was called a cluster zero-temperature process (CZTP). Here $\mathbf{c}$ is a set of pixels, and we set $\mathbf{c}=\{(i, j),(i+1, j),(i+1, j+1),(i, j+1)\}$ in our numerical experiments in Sec. 7.

\section{Quantum Mean-field Iterative Algorithm}

In this section, we extend the quantum mean-field iterative algorithm [19] for binary images to images with $q$ grades.

In order to investigate the quantum effect on the search of the energy minimum configuration, we introduce a matrix for the energies as

$$
\left\langle\mathbf{x}\left|\hat{\mathbf{H}}_{\mathrm{C}}\right| \mathbf{x}^{\prime}\right\rangle \equiv H\left(\mathbf{x} \mid \mathbf{y}^{*}, \mathbf{J}^{*}\right) \prod_{i=1}^{M} \prod_{j=1}^{N} \delta\left(x_{i, j}, x_{i, j}^{\prime}\right),
$$

where $|\mathbf{x}\rangle$ is a $q^{M N}$-component vector defined by the direct product of the $M N$ vectors $\left|x_{i, j}\right\rangle$ as

$$
|\mathbf{x}\rangle=\prod_{(i, j) \in \mathbf{I}} \otimes\left|x_{i, j}\right\rangle
$$

The $n$-th component of the vector $\left|x_{i, j}\right\rangle$ is defined as

$$
\left|x_{i, j}\right\rangle_{n} \equiv \delta\left(x_{i, j}, n\right)
$$

At this stage, the search of the energy minimum configuration is replaced with that of the eigenvector which corresponds to the minimum eigenvalue of the hamiltonian matrix $\hat{\mathbf{H}}_{\mathrm{C}}$. As a basic quantum effect, we introduce the vector field $-h \sum_{(i, j) \in \mathbf{I}} \hat{\mathbf{S}}_{i, j}^{q}$ and define the following hamiltonian

$$
\hat{\mathbf{H}} \equiv \hat{\mathbf{H}}_{\mathrm{C}}-\sum_{(i, j) \in \mathbf{I}} \hat{\mathbf{s}}_{i, j}^{q}
$$




$$
\begin{aligned}
= & \sum_{(i, j) \in \mathbf{I}}\left[-h \hat{\mathbf{s}}_{i, j}^{q}+\sum_{m=0}^{q-1}\left\{-\delta\left(m, y_{i, j}\right) \hat{\mathbf{s}}_{i, j}^{m}+\sum_{n=1}^{k-1} J_{n}^{*} \sum_{\left(i^{\prime}, j^{\prime}\right) \in \mathbf{c}_{i, j}}\left(\hat{\mathbf{s}}_{i, j}^{m} \hat{\mathbf{s}}_{i+1, j}^{m+n}+\hat{\mathbf{s}}_{i, j}^{m+n} \hat{\mathbf{s}}_{i+1, j}^{m}+\hat{\mathbf{s}}_{i, j}^{m} \hat{\mathbf{s}}_{i, j+1}^{m+n}+\hat{\mathbf{s}}_{i, j}^{m+n} \hat{\mathbf{s}}_{i, j+1}^{m}\right)\right.\right. \\
& \left.\left.+J_{k}^{*} \sum_{n=k}^{q-m-1} \sum_{\left(i^{\prime}, j^{\prime}\right) \in \mathbf{c}_{i, j}}\left(\hat{\mathbf{s}}_{i, j}^{m} \hat{\mathbf{s}}_{i+1, j}^{m+n}+\hat{\mathbf{s}}_{i, j}^{m+n} \hat{\mathbf{s}}_{i+\mathbf{1}, j}^{m}+\hat{\mathbf{s}}_{i, j}^{m} \hat{\mathbf{s}}_{i, j+\mathbf{1}}^{m+n}+\hat{\mathbf{s}}_{i, j}^{m+n} \hat{\mathbf{s}}_{i, j+1}^{m}\right)\right\}\right],
\end{aligned}
$$

where

$$
\begin{gathered}
\hat{\mathbf{s}}_{i, j}^{m} \equiv \stackrel{(1,1)}{\hat{\mathbf{e}}} \otimes \cdots \otimes \hat{\mathbf{e}} \otimes \stackrel{(i, j)}{\hat{\mathbf{s}}^{m}} \otimes \hat{\mathbf{e}} \cdots \otimes \stackrel{(M, N)}{\hat{\mathbf{e}}}, \\
\hat{\mathbf{s}}_{i, j}^{q} \equiv \stackrel{(1,1)}{\hat{\mathbf{e}}} \otimes \cdots \otimes \hat{\mathbf{e}} \otimes \stackrel{(i, j)}{\mathbf{\hat { \mathbf { s } }}}{ }^{q} \otimes \hat{\mathbf{e}} \otimes \cdots \otimes \stackrel{(M, N)}{\hat{\mathbf{e}}},
\end{gathered}
$$

and the $\left(n, n^{\prime}\right)$ element of the $q \times q$ matrix $\hat{\mathbf{s}}^{m}$ and that of $\hat{\mathbf{s}}^{q}$ are given by

$$
\begin{aligned}
& \left\langle n\left|\hat{\mathbf{s}}^{m}\right| n^{\prime}\right\rangle \equiv \delta\left(n, n^{\prime}\right) \delta(m, n) \quad(m \in \mathbf{Q}), \\
& \left\langle n\left|\hat{\mathbf{s}}^{q}\right| n^{\prime}\right\rangle \equiv 1-\delta\left(n, n^{\prime}\right) .
\end{aligned}
$$

If we express the minimum eigenvalue and its corresponding right eigenvector of the hamiltonian matrix $\hat{\mathbf{H}}$ by $\lambda$ and $|\phi\rangle$, respectively, we have

$$
\left.|\phi\rangle=\arg \min _{|\Phi\rangle}\{\lambda|\hat{\mathbf{H}}| \Phi\rangle=\lambda|\Phi\rangle\right\}
$$

If we put $h=0$, Eq. (69) can be reduced to Eq. (12).

In order to adopt the mean-field approximation for the search of the eigenvector $|\phi\rangle$, which corresponds to the minimum eigenvalue $\lambda$, we introduce a $q$-component vector $\left|\phi_{i, j}\right\rangle$ from the vector $|\phi\rangle$ as follows:

$$
\left|\phi_{i, j}\right\rangle\left\langle\phi_{i, j}\right| \equiv\left\langle\phi\left|\hat{\mathbf{s}}_{i, j}^{q}\right| \phi\right\rangle \hat{\mathbf{s}}^{x}+\sum_{m=0}^{q-1}\left\langle\phi\left|\hat{\mathbf{s}}_{i, j}^{m}\right| \phi\right\rangle \hat{\mathbf{s}}^{m} .
$$

The vector $\left|\phi_{i, j}\right\rangle$ should satisfy the following equality:

$$
\left\langle\phi_{i, j} \mid \phi_{i, j}\right\rangle=1 \text {. }
$$

The Eq. (69) is then reduced to

$$
\left.\left|\phi_{i, j}\right\rangle=\arg \min _{|\Phi\rangle}\left\{\lambda_{i, j}\left|\lambda_{i, j}=\langle\Phi| \hat{\mathbf{H}}_{i, j}\left(\left|\phi_{i^{\prime}, j^{\prime}}\right\rangle,\left(i^{\prime}, j^{\prime}\right) \in \mathbf{I} \backslash(i, j)\right)\right| \Phi\right\rangle\right\},
$$

where $\left(n, n^{\prime}\right)$-component of $q \times q$ matrix $\hat{\mathbf{H}}_{i, j}$ is defined by

$$
\begin{aligned}
& \langle n| \hat{\mathbf{H}}_{i, j}\left(\left|\phi_{i^{\prime}, j^{\prime}}\right\rangle,\left(i^{\prime}, j^{\prime}\right) \in \mathbf{I} \backslash(i, j)\right)\left|n^{\prime}\right\rangle \\
& \equiv\left[\prod_{\left.i^{\prime}, j^{\prime}\right) \in \mathbf{I}} \otimes\left(\left(1-\delta\left(i, i^{\prime}\right) \delta\left(j, j^{\prime}\right)\right)\left\langle\phi_{i^{\prime}, j^{\prime}}\right|+\delta\left(i, i^{\prime}\right) \delta\left(j, j^{\prime}\right)\left\langle n^{\prime}\right|\right)\right] \\
& \quad \times \hat{\mathbf{H}}\left[\prod_{\left(i^{\prime}, j^{\prime}\right) \in \mathbf{I}} \otimes\left(\left(1-\delta\left(i, i^{\prime}\right) \delta\left(j, j^{\prime}\right)\right)\left|\phi_{i^{\prime}, j^{\prime}}\right\rangle+\delta(i, j)|n\rangle\right)\right] \quad\left(n, n^{\prime} \in \mathbf{Q}\right) .
\end{aligned}
$$

By substituting Eq. (64) into Eq. (73), the $q \times q$ matrix $\hat{\mathbf{H}}_{i, j}$ is expressed by

$$
\begin{aligned}
\hat{\mathbf{H}}_{i, j}\left(\left|\phi_{i^{\prime}, j^{\prime}}\right\rangle,\left(i^{\prime}, j^{\prime}\right) \in \mathbf{I} \backslash(i, j)\right) \equiv & -h \hat{\mathbf{s}}^{q}+\sum_{m=0}^{q-1} \hat{\mathbf{s}}^{m}\left[-\delta\left(m, y_{i, j}\right)\right. \\
& +\sum_{\left.i^{\prime}, j^{\prime}\right) \in \mathbf{c}_{i, j}} \sum_{n=1}^{k-1} J_{n}^{*}\left(\left\langle\phi_{i^{\prime}, j^{\prime}}\left|\hat{\mathbf{s}}^{m+n}\right| \phi_{i^{\prime}, j^{\prime}}\right\rangle+\left\langle\phi_{i^{\prime}, j^{\prime}}\left|\hat{\mathbf{s}}^{m-n}\right| \phi_{i^{\prime}, j^{\prime}}\right\rangle\right) \\
& \left.+\sum_{\left(i^{\prime}, j^{\prime}\right) \in \mathbf{c}_{i, j}} J_{k}^{*} \sum_{n=k}^{q-1}\left(\left\langle\phi_{i^{\prime}, j^{\prime}}\left|\hat{\mathbf{s}}^{m+n}\right| \phi_{i^{\prime}, j^{\prime}}\right\rangle+\left\langle\phi_{i^{\prime}, j^{\prime}}\left|\hat{\mathbf{s}}^{m-n}\right| \phi_{i^{\prime}, j^{\prime}}\right\rangle\right)\right],
\end{aligned}
$$

where

$$
\left\langle n\left|\hat{\mathbf{s}}^{m}\right| n^{\prime}\right\rangle \equiv 0 \quad(m \notin \mathbf{Q}) .
$$

Here the notation $\mathbf{c}_{i, j}$ is defined by Eq. (29). When a set of values of $J_{1}^{*}, J_{2}^{*}$ and $h$ is given, this simultaneous nonlinear equations for $\left\{\left|\phi_{i, j}\right\rangle\right\}$ are numerically solved by using an iteration method. We obtain $\mathbf{x}\left(\mathbf{J}^{*}\left(p^{*}, \mathbf{K}^{*}\right)\right)$ from

$$
z_{i, j}^{*}=\arg \max _{m \in \mathbf{Q}}\left\langle\phi_{i, j}\left|\hat{\mathbf{s}}^{m}\right| \phi_{i, j}\right\rangle \text {, }
$$

for small $h(>0)$. In order to solve the simultaneous non-linear equations at an enough small value of $h$ for the set of values of parameters $J_{1}^{*}$ and $J_{2}^{*}$, we start it from a sufficient large value of $h$. In Ref. [19], we solved the simultaneous non-linear equations for the next smaller value of $h$, by adopting the set of the solutions obtained for the 
last value of $h$ as the initial value in the iteration process for $q=2$. In the same way, we reduce the value of $h$ to the final small value of $h$. However, because our purpose is to obtain the image $\mathbf{z}^{*}$ for an enough small value of $h$, we introduce the transverse field in order to avoid that the iteration process is trapped to local minimum configurations; we adopt $2.5 J_{k}^{*} \ln 2 / \ln (r+1)(r=1,2, \cdots, R)$ as a schedule of $h$. We summarize our algorithm as follows:

\section{QMFI algorithm}

Step 1: Initialize so that $\left|\phi_{i, j}\right\rangle \leftarrow\left|y_{i, j}\right\rangle$ and $r \leftarrow 0$.

Step 2: Update the value of $r$ to $r+1$ and set $h \leftarrow 2.5 J_{k}^{*} \ln 2 / \ln (r+1)$. For each site $(i, j) \in \mathbf{I}$, update $\left|\phi_{i, j}\right\rangle$ according to the equation

$$
\left.\left|\phi_{i, j}\right\rangle \leftarrow \arg \min _{\left|\Phi_{i, j}\right\rangle}\left\{\lambda_{i, j}\left|\lambda_{i, j}=\left\langle\Phi_{i, j}\right| \hat{\mathbf{H}}_{i, j}\left(\left|\phi_{i^{\prime}, j^{\prime}}\right\rangle,\left(i^{\prime}, j^{\prime}\right) \in \mathbf{I} \backslash(i, j)\right)\right| \Phi_{i, j}\right\rangle\right\} .
$$

Step 3: If $r=R$, then

$$
\left.z_{i, j}^{*} \leftarrow \arg \max _{m \in \mathbf{Q}}\left\langle\phi_{i, j}\left|\hat{\mathbf{s}}^{m}\right| \phi_{i, j}\right\rangle\right)
$$

and stop, else go to Step 2.

\section{Practical Energy Function for Real-World Images}

In the previous sections, we have given the energy function for image restorations of the gray level images, described a method for the determination of its hyperparameters by means of the maximum likelihood estimation and the optimization techniques of the energy function. However, by using this energy function, there are some problems as for the search of the optimal configuration $\mathbf{z}^{*}$ by using the probabilistic computational algorithm, the cluster zero-temperature algorithm or the quantum iterated conditional algorithm given in Secs. 3, 4 and 5 , respectively. Moreover, we have to estimate a lot of hyperparameters in the real-world digital images with 256 grades. Practically, the formulation in the previous sections is useful for images with low grades. For example, in the case of $q=8$, when we assume $k=2$, all the hyperparameters to be estimated are only $K_{1}^{*}$ and $K_{2}^{*}$. However, in the case of $q=256$, when we assume $32 \leq k \leq 64$, we have to estimate more than 30 hyperparameters. This is practically impossible from the computational time and memory at the present time. In order to avoid this difficulty, we impose an additional assumption upon the form of $K_{n}^{*}(n=1,2, \cdots, k)$ in the energy function given in Eq. (10) with Eqs. (8) and (11). Namely, we assume the following form of $n$-dependence:

$$
K_{n}^{*}=T\left(\tilde{p}^{*}\right) \times \min \left\{\tilde{K}_{1}^{*}\left(\frac{n}{32}\right)^{2}, \tilde{K}_{2}^{*}\right\} \quad(n=1,2, \cdots, k), \quad \tilde{p}^{*} \equiv \frac{7}{8} p^{*},
$$

where $\tilde{K}_{1}^{*}$ and $\tilde{K}_{2}^{*}$ are estimated by using Eqs. (16) and (17) with Eq. (3) for $q=8$. The deterministic equations of $\tilde{K}_{1}^{*}$ and $\tilde{K}_{2}^{*}$ are given as follows:

$$
\begin{gathered}
\sum_{\mathbf{x} \in \tilde{\mathbf{Q}}^{M N}} \sigma_{1}(\mathbf{x}) \operatorname{Pr}\left\{\mathbf{X}=\mathbf{x} \mid \tilde{K}_{1}^{*}, \tilde{K}_{2}^{*}\right\}=\sigma_{1}\left(\tilde{\mathbf{x}}^{*}\right), \\
\sum_{\mathbf{x} \in \tilde{\mathbf{Q}}^{M N}}\left(\sum_{n=2}^{7} \sigma_{n}(\mathbf{x})\right) \operatorname{Pr}\left\{\mathbf{X}=\mathbf{x} \mid \tilde{K}_{1}^{*}, \tilde{K}_{2}^{*}\right\}=\sum_{n=2}^{7} \sigma_{n}\left(\tilde{\mathbf{x}}^{*}\right), \\
\tilde{\mathbf{x}}^{*} \equiv\left\{\tilde{x}_{i, j}^{*} \mid \tilde{x}_{i, j}^{*}=\operatorname{int}\left(\frac{x_{i, j}^{*}}{32}\right), \quad(i, j) \in \mathbf{I}\right\},
\end{gathered}
$$

where $\operatorname{Pr}\left\{\mathbf{X}=\mathbf{x} \mid \tilde{K}_{1}^{*}, \tilde{K}_{2}^{*}\right\}$ is the a priori probability distribution given in Eq. (3) for $q=8$. This assumption means that the a priori probability distribution is estimated from the images $\tilde{\mathbf{x}}^{*}$ with 8 grades obtained from the image $\mathbf{x}^{*}$ with 256 grades by using Eq. (80). The optimal configuration $\mathbf{z}^{*}$ of the energy function given in Eq. (10) for $q=8$ with Eq. (79) is obtained by using the ICM algorithm given in Sec. 4.

In the ICM algorithm, it is important how we choose an initial configuration $\mathbf{z}^{(0)}$. The simplest choice is $\mathbf{z}^{(0)}=$ y. However, in this choice, we think that the iteration might be trapped in some local minimum configuration. In order to avoid such a trouble, we set an initial image $\mathbf{z}^{(0)}$ to $z_{i, j}^{(0)}=$ int $\left(32 z_{i, j}^{*}+16\right)$; the image $\tilde{\mathbf{z}}^{*}$ is the optimal configuration of the energy function (10) in the case of $q=8$. By this choice, we can expect to obtain a nearer local minimum configuration to the true optimal configuration than the local minimum configuration obtained by choosing the degraded image $\mathbf{y}$ as the initial configuration $\mathbf{z}^{(0)}$.

\section{Numerical Experiments}

In this section, we give some results by numerical experiments for the original image $\mathbf{x}^{*}$ given in Fig. 1 for the cases of $q=8$ and $q=256$. Both images in Figs. 1(c) and 1(d) have 256 grades and are standard images which 
(a)

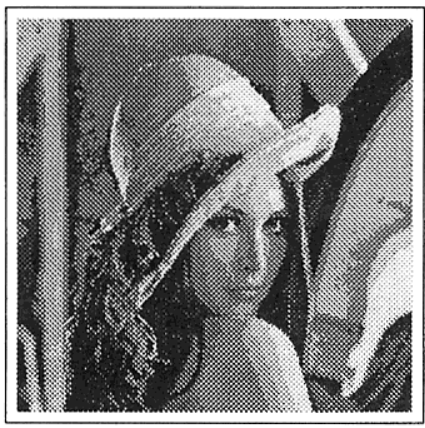

(c)

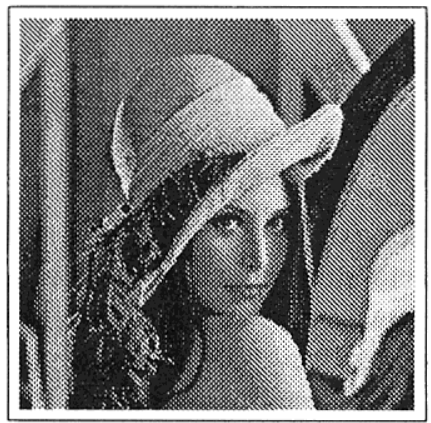

(b)

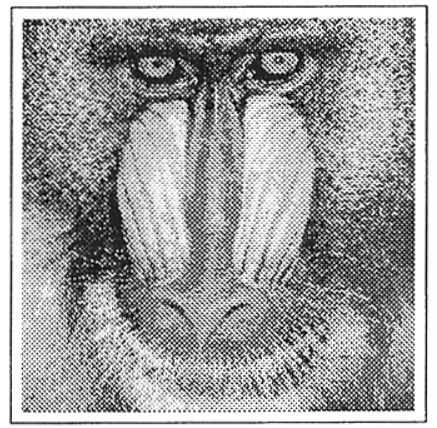

(d)

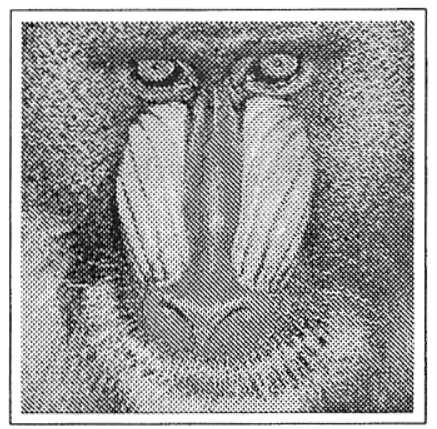

Fig. 1. Original images $\mathbf{x}^{*}(M=N=256)$. (a) Lena $(q=8)$. (b) Mandrill $(q=8)$. (c) Lena $(q=256)$. (d) Mandrill ( $q$ = 256).

Table 1: Values of $\bar{\sigma}_{n}\left(\mathbf{x}^{*}\right)$ for the true original image $\mathbf{x}^{*}$ given in Figs. 1(a) and 1(b).

\begin{tabular}{|c|c|c|c|c|c|c|c|c|c|}
\hline Figure & $\bar{\sigma}_{0}\left(\mathbf{x}^{*}\right)$ & $\bar{\sigma}_{1}\left(\mathbf{x}^{*}\right)$ & $\bar{\sigma}_{2}\left(\mathbf{x}^{*}\right)$ & $\bar{\sigma}_{3}\left(\mathbf{x}^{*}\right)$ & $\bar{\sigma}_{4}\left(\mathbf{x}^{*}\right)$ & $\bar{\sigma}_{5}\left(\mathbf{x}^{*}\right)$ & $\bar{\sigma}_{6}\left(\mathbf{x}^{*}\right)$ \\
\hline $1(\mathrm{a})$ & 0.76308 & 0.20512 & 0.02159 & 0.00601 & 0.00206 & 0.00092 & 0.00063 & 0.00058 \\
\hline $1(\mathrm{~b})$ & 0.53013 & 0.36436 & 0.08249 & 0.01699 & 0.00341 & 0.00182 & 0.00076 \\
\hline
\end{tabular}

are adopted for numerical experiments by many authors in image processing. The images in Figs. 1(a) and 1(b) are obtained by the transformation $\tilde{x}_{i, j}^{*}=$ int $\left(x_{i, j}^{*} / 32\right)$ from the images $\mathbf{x}^{*}$ in Figs. $1(\mathrm{c})$ and $1(\mathrm{~d})$. We set $M=N$ $=256$.

First we do some numerical experiments for $q=8$ when the images given in Figs. 1(a) and 1(b) are adopted as an original image $\mathbf{x}^{*}$. The degradation process is subject to the probability given in Eq. (1) for $q=8$. Here, we set $(q-1) p^{*}=0.1,0.2,0.3,0.4$ and 0.5 . For $(q-1) p^{*}=0.3$, the degraded images $\mathbf{y}^{*}$ obtained from the original image $\mathbf{x}^{*}$ in Figs. 1(a) and 1(b) are given in Figs. 2(a) and 3(a), respectively. The quantities $\bar{\sigma}_{n}\left(\mathbf{x}^{*}\right) \equiv(1 /$ $2 M N) \sigma_{n}\left(\mathbf{x}^{*}\right)(n \in \mathbf{Q})$ are shown in Table 1. We see that the quantities $\sigma_{0}\left(\mathbf{x}^{*}\right)$ and $\sigma_{1}\left(\mathbf{x}^{*}\right)$ are especially important in images with 8 grades, and hence we set $k=2$ in the energy function given in Eqs. (10), (11) and (64). Values of hyperparameters $K_{1}^{*}$ and $K_{2}^{*}$, are estimated by means of the maximum likelihood estimation with the pair approximation. The deterministic equations in the maximum likelihood estimation of $K_{1}^{*}$ and $K_{2}^{*}$ are given by Eqs. (55), (56), (57) and (58). For $(q-1) p^{*}=0.3$, we show the restored images $\mathbf{z}^{*}$ obtained by using the MFA, the PA, the ICM, the CZTP and the QMFI algorithm at the values of hyperparameters $K_{1}^{*}$ and $K_{2}^{*}$ in Figs. 2(b)-(f) and 3(b)-(f), respectively. In the CZTP algorithm, we set $\mathbf{c} \equiv\{(i, j),(i+1, j),(i+1, j+1),(i, j+1)\}$. In Table 2, we give the quantities $\vec{d}\left(\mathbf{z}^{*}, \mathbf{x}^{*}\right) \equiv(1 / M N) d\left(\mathbf{z}^{*}, \mathbf{x}^{*}\right)$ for the restored image $\mathbf{z}^{*}$, which are obtained for each values of $(q-1) p^{*}$ by using the MFA, the PA, the ICM, the CZTP and the QMFI.

Second, we give some results for the case of $q=256$. The original images $\mathbf{x}^{*}$ treated in this case are given in Figs. 1(c) and 1(d). We adopt $H\left(\mathbf{x} \mid \mathbf{y}^{*}, \mathbf{J}^{*}\right)$ defined by Eq. (10) with Eqs. (8), (11) and (77) as the energy function for $q=256$. The values of hyperparameters $\tilde{K}_{1}$ and $\tilde{K}_{2}$ in Eq. (77) are determined by applying Eqs. (78), (79) and $(80)$ to the original images $\mathbf{x}^{*}$ given in Figs. $1(\mathrm{c})$ and $1(\mathrm{~d})$. The degradation process is subject to the probability $\operatorname{Pr}\left\{\mathbf{Y}=\mathbf{y} \mid \mathbf{X}=\mathbf{x}^{*}, p^{*}\right\}$ given in Eq. (1) for $q=256$. Here, we set $(q-1) p^{*}=0.3$ and 0.5 . For $(q-1) p^{*}=$ 0.3 , the degraded images $\mathbf{y}^{*}$ obtained from the original image $\mathbf{x}^{*}$ in Figs. 1(c) and 1(d) are given in Figs. 4(a) and 
(a)

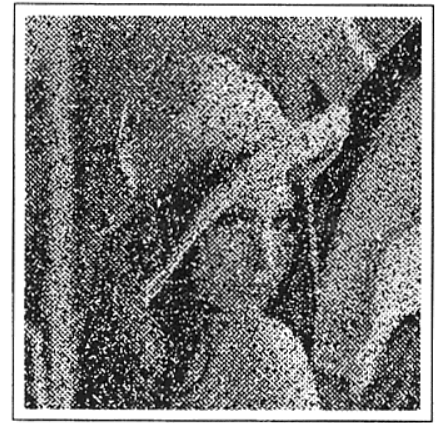

(c)

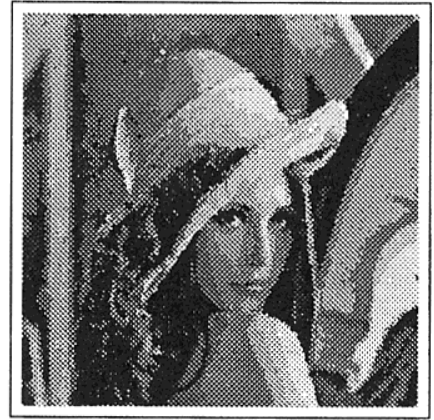

(e)

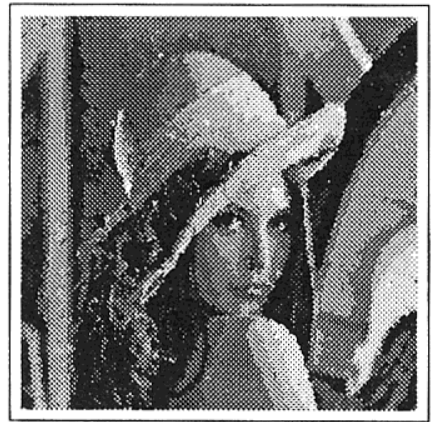

(b)

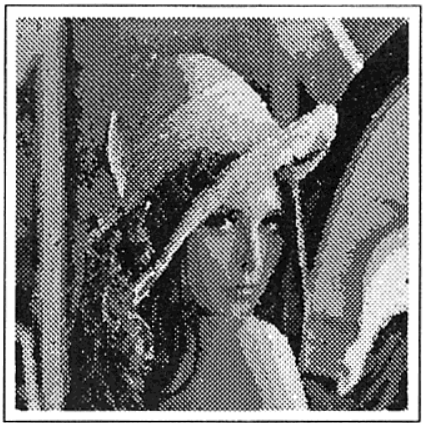

(d)

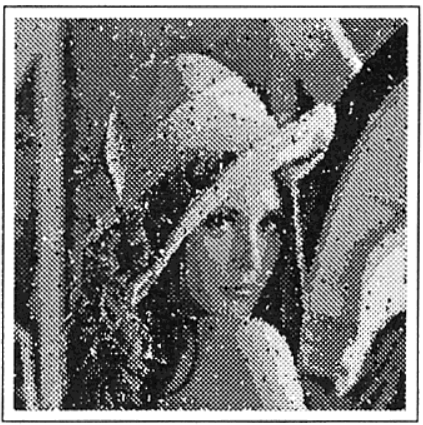

(f)

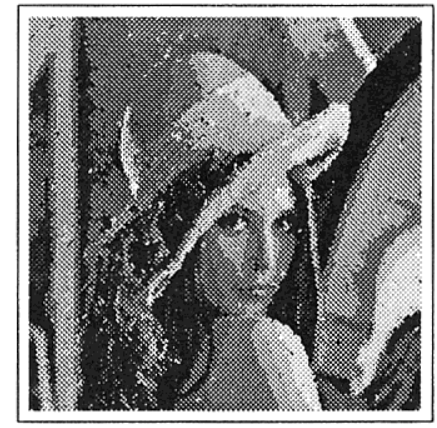

Fig. 2. Restored images $\mathbf{z}^{*}$ for the original images $\mathbf{x}^{*}$ given in Fig. 1(a). (a) Degraded image $\mathbf{y}^{*}\left(q=8,(q-1) p^{*}=\right.$ 0.3). (b) MFA $z^{*}$. (c) PA $z^{*}$. (d) ICM $z^{*}$. (e) CZTP $z^{*}$. (f) QMFA $z^{*}$.

Table 2: Values of $\bar{d}\left(\mathbf{x}^{*}, \mathbf{z}^{*}\right)$ for gray-level image restorations with 8 grades $(q=8)$. The degraded images $\mathbf{y}^{*}$ are produced by using the degradation process (1) from the original image $\mathbf{x}^{*}$ given in Figs. 1 (a) and $1(\mathrm{~b})$. The restored images $\mathbf{z}^{*}$ are obtained by using the ICM, the CZTP, the QMFI, the MFA and the PA algorithm at the values of hyperparameters $K_{1}^{*}$ and $K_{2}^{*}$.

(a) Lena $\left(K_{1}^{*} \simeq 0.8658, K_{2}^{*} \simeq 1.7381\right)$.

\begin{tabular}{|l|c|c|c|c|c|}
\hline Optimization & $(q-1) p^{*}=0.1$ & $(q-1) p^{*}=0.2$ & $(q-1) p^{*}=0.3$ & $(q-1) p^{*}=0.4$ & $(q-1) p^{*}=0.5$ \\
\hline ICM & 0.0470 & 0.0788 & 0.1289 & 0.1689 & 0.2468 \\
CZTP & 0.0437 & 0.0730 & 0.1081 & 0.1411 & 0.1875 \\
MFA & 0.0426 & 0.0688 & 0.1033 & 0.1333 & 0.1726 \\
PA & 0.0427 & 0.0690 & 0.1023 & 0.1315 & 0.1718 \\
QMFI & 0.0440 & 0.0721 & 0.1062 & 0.1410 & 0.1843 \\
\hline
\end{tabular}

(b) $\operatorname{Mandrill~}\left(K_{1}^{*} \simeq 0.64889, K_{2}^{*} \simeq 1.55443\right)$.

\begin{tabular}{|l|c|c|c|c|c|}
\hline Optimization & $(q-1) p^{*}=0.1$ & $(q-1) p^{*}=0.2$ & $(q-1) p^{*}=0.3$ & $(q-1) p^{*}=0.4$ & $(q-1) p^{*}=0.5$ \\
\hline ICM & 0.0751 & 0.1497 & 0.2231 & 0.2932 & 0.3729 \\
CZTP & 0.0731 & 0.1430 & 0.2153 & 0.2841 & 0.3567 \\
MFA & 0.0717 & 0.1413 & 0.2091 & 0.2762 & 0.3457 \\
PA & 0.0718 & 0.1412 & 0.2069 & 0.2716 & 0.3402 \\
QMFI & 0.0731 & 0.1417 & 0.2134 & 0.2795 & 0.3456 \\
\hline
\end{tabular}


(a)

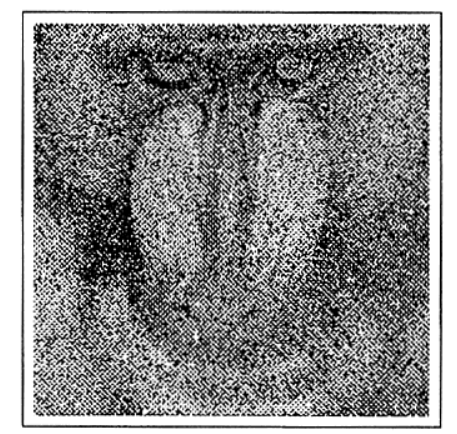

(c)

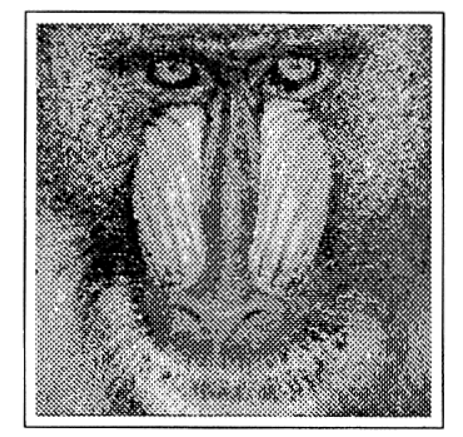

(e)

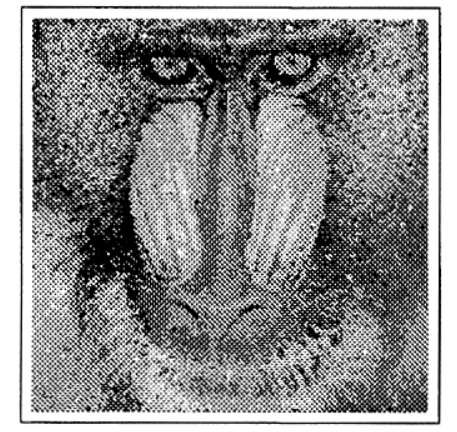

(b)

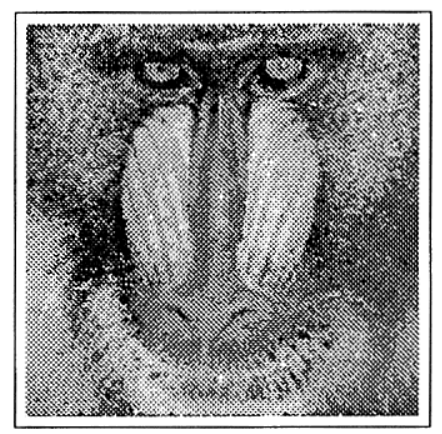

(d)

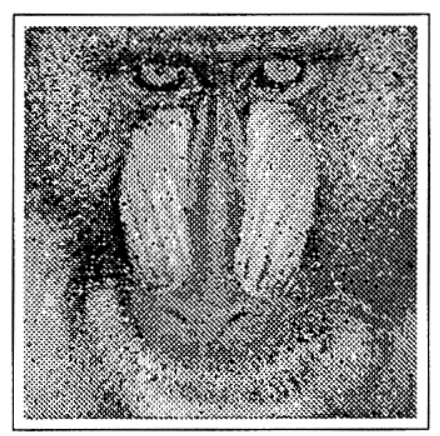

(f)

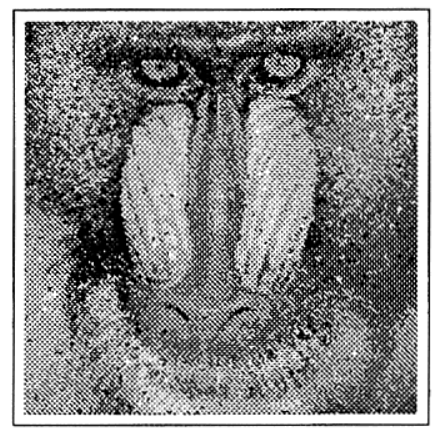

Fig. 3. Restored image $\mathbf{z}^{*}$ for the original image $\mathbf{x}^{*}$ given in Fig. 1(b). (a) Degraded image $\mathbf{y}^{*}\left(q=8,(q-1) p^{*}=0.3\right)$. (b) MFA $\mathbf{z}^{*}$. (c) PA $\mathbf{z}^{*}$. (d) ICM $\mathbf{z}^{*}$. (e) CZTP $\mathbf{z}^{*}$. (f) QMFA $\mathbf{z}^{*}$.

5(a), respectively. The degraded images $\mathbf{y}^{*}$ are reduced to the corresponding 8-valued images $\tilde{\mathbf{y}} \equiv\left\{\tilde{y}_{i, j} \mid \tilde{y}_{i, j} \equiv\right.$ int $\left.\left(y_{i, j} / 32\right),(i, j) \in \mathbf{I}\right\}$. We obtain the restored images $\tilde{\mathbf{z}}^{*}$ by means of the MFA, the PA, the ICM, the CZTP and the QMFI algorithm for $q=8$, for the case that the reduced images $\tilde{\mathbf{y}}$ are regarded as the 8-valued degraded images. We also obtain the restored images $\mathbf{z}^{*}$ for $q=256$ by setting $\mathbf{z}^{(0)} \equiv\left\{z_{\mathrm{i}, j}^{(0)} \mid z_{i, j}^{(0)} \equiv 32 \tilde{z}_{i, j}^{*}+16\right\}$ from the restored images $\tilde{\mathbf{z}}^{*}$ for $q=8$, and by applying the ICM for the energy function given in Eq. (10) with Eqs. (8), (11) and (77) for the case of $q=256$ to the degraded image $\mathbf{y}^{*}$. The mean square errors $\bar{d}_{\mathrm{MSE}}\left(\mathbf{x}^{*}, \mathbf{z}^{*}\right) \equiv(1 / M N)$ $\Sigma_{(i, j) \in \mathbf{I}}\left(x_{i, j}^{*}-z_{i, j}^{*}\right)^{2}$ for the restored images $\mathbf{z}^{*}$ by means of these algorithms are given in Table 3 . The restored images $\mathbf{z}^{*}$ for $(q-1) p^{*}=0.3$ are given in Figs. 4(b)-(f) and 5(b)-(f).

The results obtained in these numerical experiments for both cases of $q=8$ and $q=256$ show that the MFA and the PA algorithm gives the images which are closer to the original images than those obtained by means of not only the ICM algorithm but also the CZTP and the QMFI algorithm in the stage of 8 grades.

\section{Concluding Remarks}

We summarize the contents of the present paper as for image restorations by means of the MRF model from the standpoint of the maximum likelihood estimation [2], [3] and some optimization algorithms based on the statistical-mechanical techniques for the energy minimization problem formulated for gray-level image restora- 
(a)

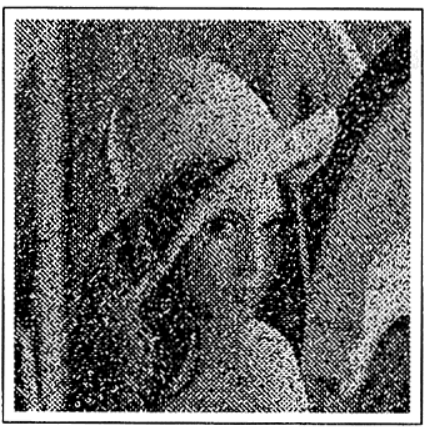

(c)

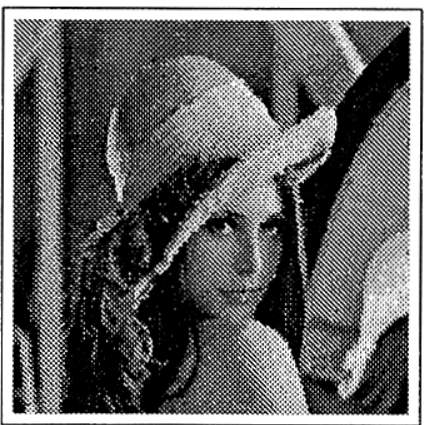

(e)

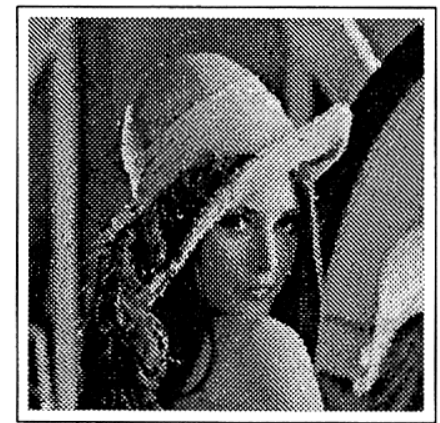

(b)

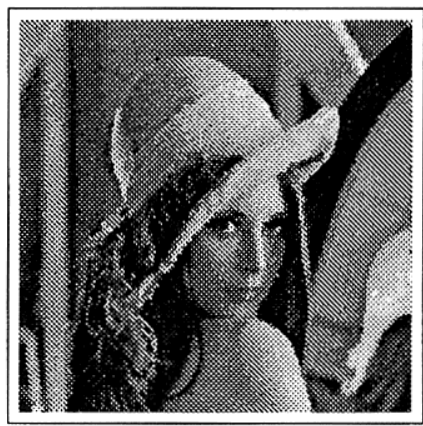

(d)

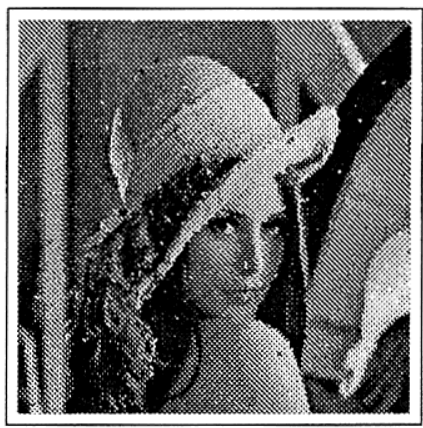

(f)

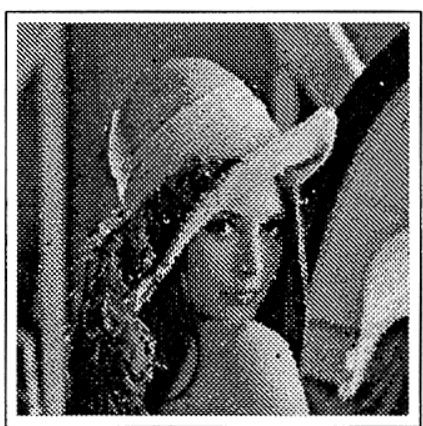

Fig. 4. Restored images $\mathbf{z}^{*}$ from the original image $\mathbf{x}^{*}$ given in Fig. 1(c). (a) Degraded image $\mathbf{y}^{*}\left(q=256,(q-1) p^{*}=\right.$ $0.3)$. (b) MFA $(q=8) \rightarrow \operatorname{ICM}(q=256) \mathbf{z}^{*}$. (c) PA $(q=8) \rightarrow \operatorname{ICM}(q=256) \mathbf{z}^{*}$. (d) $\operatorname{ICM}(q=8) \rightarrow \operatorname{ICM}(q=$ 256) $\mathbf{z}^{*}$. (e) CZTP $(q=8) \rightarrow \operatorname{ICM}(q=256) \mathbf{z}^{*}$. (f) QMFA $(q=8) \rightarrow \operatorname{ICM}(q=256) \mathbf{z}^{*}$.

tions. The main results are that the MFA and the PA algorithm give the restored images closer to the original image than those obtained by the ICM, the CZTP and the QMFI algorithm. This fact means that the thermal fluctuation is the most important in the optimization techniques. It means that just the thermal fluctuation is not important but it is important if the hyperparameters pass through near the values specified by Nishimori-Wong's inequality in the annealing process. Nishimori and Wong [7] proved Nishimori-Wong's inequality (19) only for the case of $q=2$. Nishimori-Wong's inequality (19) has not been proved for any positive integer $q(>2)$, yet. This is still an open problem.

In the present paper, we have adopted the maximum likelihood estimation in the a priori probability distribution to determine the values of hyperparameters. In order to clarify the relationship between the optimization algorithm and Nishimori-Wong's inequality, we use the original image $\mathbf{x}^{*}$ to estimate the hyperparameters in the maximum likelihood estimation. Many authors used the maximum marginal likelihood estimation to determine values of the hyperparameters [5]. The maximum marginal likelihood estimation can give the optimal values of the hyperparameters from only the given degraded image $\mathbf{y}^{*}$ without using the original image $\mathbf{x}^{*}$. On the other hand, we have to calculate the marginal likelihood function, $\operatorname{Pr}\left\{\mathbf{Y}=\mathbf{y}^{*} \mid p, \mathbf{K}\right\} \equiv \Sigma_{\mathbf{x} \in \mathbf{Q}^{M N}} \operatorname{Pr}\left\{\mathbf{Y}=\mathbf{y}^{*} \mid \mathbf{X}=\mathbf{x}\right.$, $p\} \operatorname{Pr}\{\mathbf{X}=\mathbf{x} \mid \mathbf{K}\}$. Pryce and Bruce calculated the marginal likelihood function by using the spin-1/2 Ising model and the mean-field approximation only for binary image restorations [5]. Recently, one of the present 
(a)

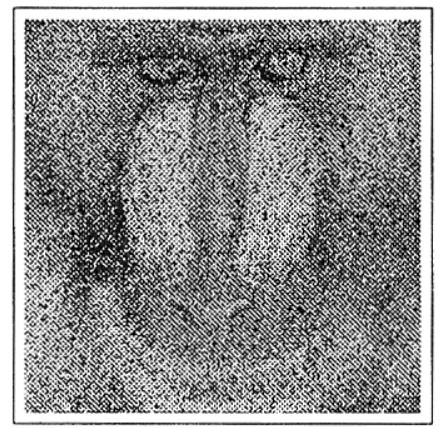

(c)

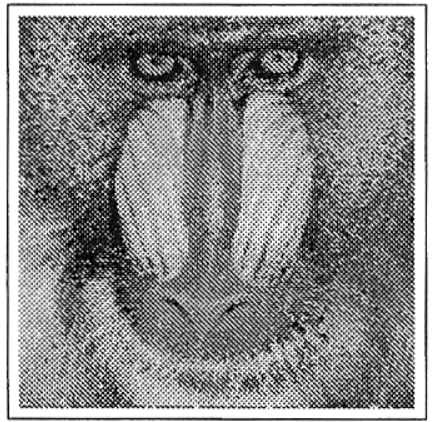

(e)

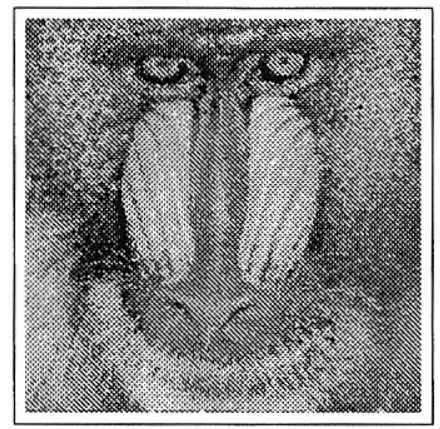

(b)

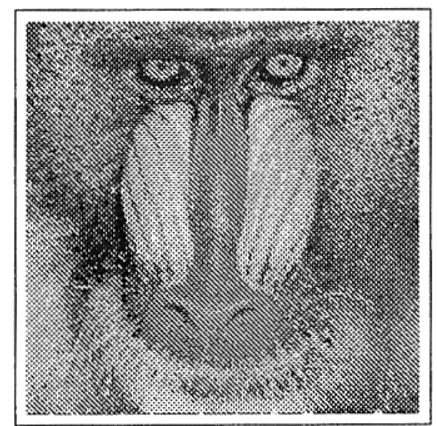

(d)

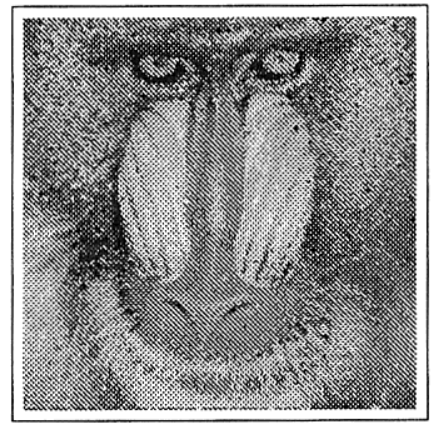

(f)

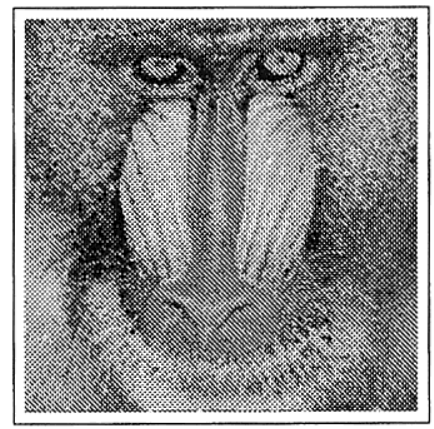

Fig. 5. Restored images $\mathbf{z}^{*}$ from the original image $\mathbf{x}^{*}$ given in Fig. 1(d). (a) Degraded image $\mathbf{y}^{*}\left(q=256,(q-1) p^{*}=\right.$ $0.3)$. (b) $\operatorname{MFA}(q=8) \rightarrow \operatorname{ICM}(q=256) \mathbf{z}^{*}$. (c) PA $(q=8) \rightarrow \operatorname{ICM}(q=256) \mathbf{z}^{*}$. (d) $\operatorname{ICM}(q=8) \rightarrow \operatorname{ICM}(q=$ 256) $\mathbf{z}^{*}$. (e) $\operatorname{CZTP}(q=8) \rightarrow \operatorname{ICM}(q=256) \mathbf{z}^{*}$. (f) QMFA $(q=8) \rightarrow \operatorname{ICM}(q=256) \mathbf{z}^{*}$.

authors (KT) has studied the maximum marginal likelihood estimates by using the $q$-state Potts model and the CVM for multi-valued image restorations [22]. It is a future problem to give a practical algorithm for determining the maximum marginal likelihood estimates for practical gray-level image restorations in high accuracy by using the CVM.

\section{REFERENCES}

[1] H. Derin, H. Elliott, R. Cristi and D. Geman, "Bayes smoothing algorithms for segmentation of binary images modeled by Markov random fields," IEEE Trans. Pattern Anal. Mach. Intell., vol. 6, no. 6, pp. 707-720, November 1984.

[2] J. Besag, “On the statistical analysis of dirty picture (with discussion)," J. Royal Statistical Society Ser. B, vol. 48, no. 3, pp. 259-302, 1986.

[3] S. Lakshmanan and H. Derin, "Simultaneous parameter estimation and segmentation of Gibbs random fields using simulated annealing," IEEE Trans. Pattern Anal. and Mach. Intel., vol. 11, no. 8, pp. 799-813, August 1989.

[4] J. Zhang, "The mean field theory in EM procedures for Markov random fields," IEEE Trans. Signal Processing, vol. 40, no. 10, pp. 2570-2583, October 1992.

[5] J. M. Pryce and A. D. Bruce, "Statistical mechanics of image restoration," Journal of Physics A, vol. 28, no. 3, pp. 511-532, February 1995. 
Table 3: Values of $\bar{d}_{\mathrm{MSE}}\left(\mathbf{x}^{*}, \mathbf{z}^{*}\right)$ in gray-level image restoration with 256 grades $(q=256)$. The restored images $\mathbf{z}^{*}$ are obtained by using the ICM algorithm for the energy function in images with 256 grades. The hyperparameters $K_{1}^{*}$ and $K_{2}^{*}$ are estimated by means of the maximum likelihood estimation for images with 8 grades. The initial configurations $\mathbf{z}^{(0)}$ for the 256 grades ICM algorithm are obtained by applying the ICM, the CZTP, the QMFI, the MFA and the PA algorithm in (a) Lena the stage of 8 grades to the reduced images $\tilde{\mathbf{y}}^{*}=\left\{\tilde{y}_{i, j}^{*}=\operatorname{int}\left(y_{i, j}^{*} / 32\right)\right\}$.

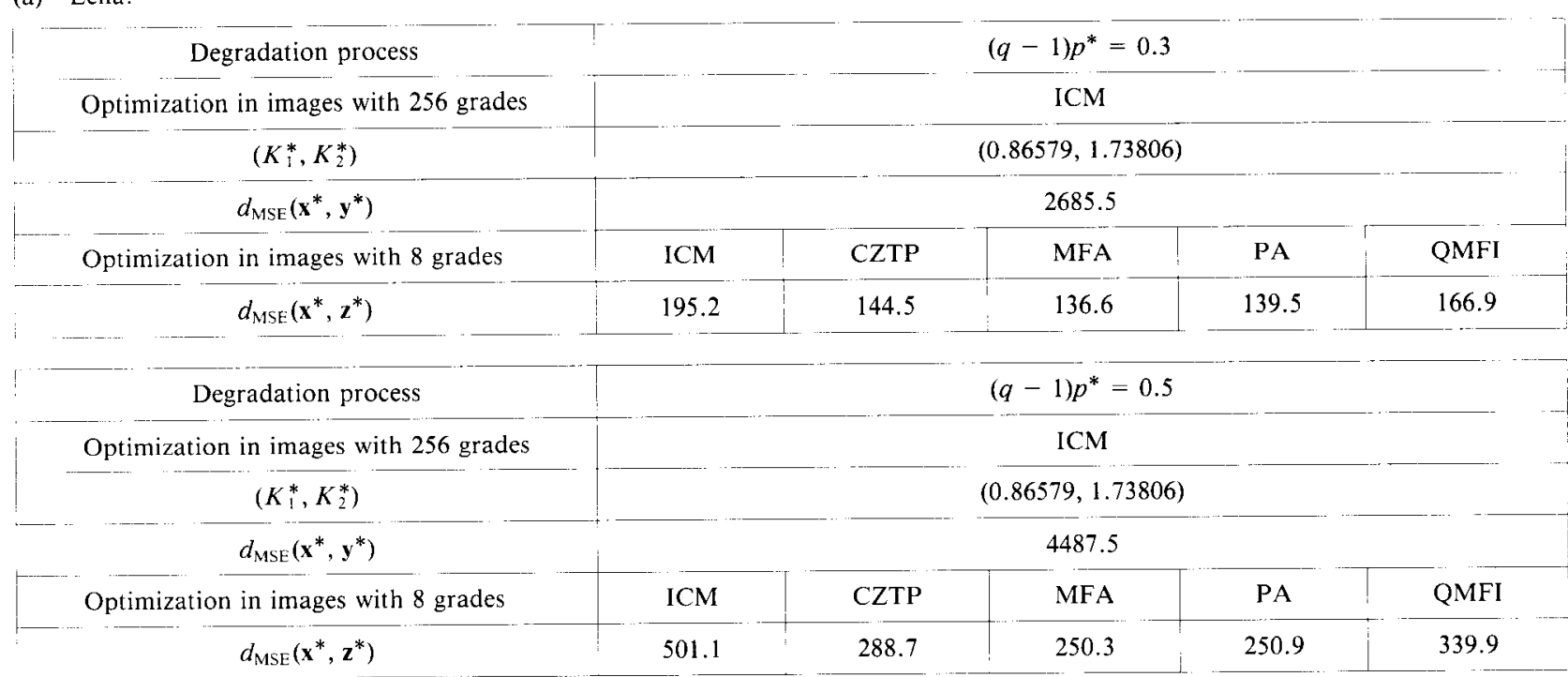

(b) Mandrill.

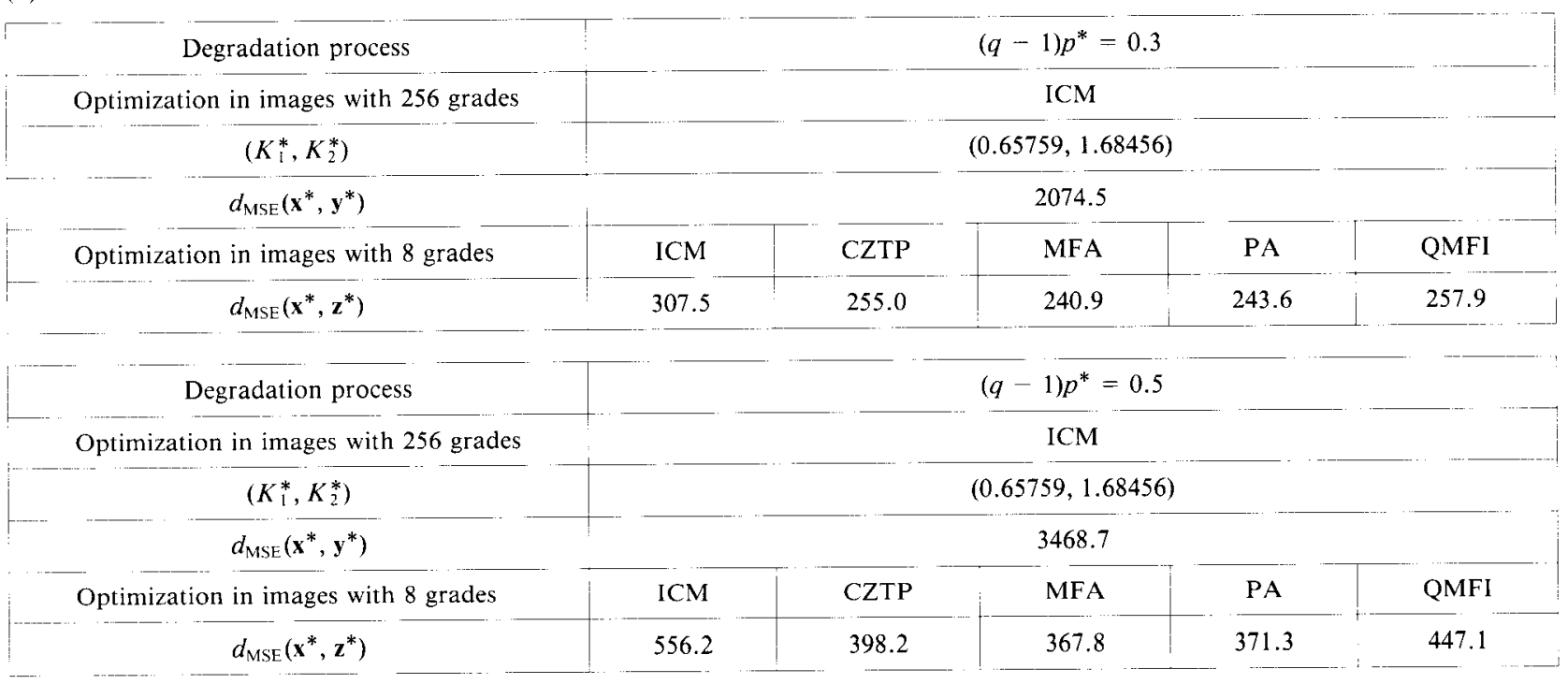

[6] J. Marroquin, S. Mitter and T. Poggio, "Probabilistic solution of ill-posed problems in computer vision," Journal of the American Statistical Association, vol. 82, no. 397, pp. 76-89, March 1987.

[7] H. Nishimori and K. Y. M. Wong, "Statistical mechanics of image restoration and error-correcting codes," Physical Review E, vol. 60, no. 1, pp. 132-144, July 1999.

[8] D. M. Carlucci and J. Inoue, "Image restoration using the chiral Potts spin glass," Physical Review E, vol. 60, no. 3, pp. 2547-2553, September 1999.

[9] T. Morita and K. Tanaka, "Determination of parameters in an image recovery of statistical-mechanical means," Physica A, vol. 223, no. 1-2, p. 244-262, 1996.

[10] K. Tanaka, M. Ichioka and T. Morita, "Statistical-mechanical iterative method in image restoration," Trans. Inst. Electron. Inf. Commun. Eng. A, vol. J80-A, no. 1, pp. 280-286, January 1997 (in Japanese).

[11] K. Tanaka, M. Ichioka and T. Morita, "Statistical-mechanical algorithm in MRF model based on variational principle," Proceedings of 13th International Conference on Pattern Recognition, vol. II, Track B: Pattern Recognition and Signal Analysis, Vienna, pp. 381-388, 1996 (IEEE Computer Society Press).

[12] K. Tanaka and T. Morita, "Statistical-mechanical iterative algorithm for image restoration of a gray level image," Trans. Inst. Electron. Inf. Commun. Eng. A, vol. J80-A, no. 6, pp. 1033-1037, June 1997 (in Japanese).

[13] D. Geiger and F. Girosi, "Parallel and deterministic algorithms from MRF's: surface reconstruction," IEEE Trans. Pattern Anal. Mach. Intell., vol. 13, no. 5, pp. 401-412, May 1991.

[14] J. Zhang, "The application of the Gibbs-Bogoliubov-Feynman inequality in mean field calculations for Markov random fields," IEEE Trans. Image Processing, vol. 5, no. 7, pp. 1208-1214, July 1996. 
[15] T. Morita, "General structure of the distribution functions for the Heisenberg model and the Ising model," J. Math. Phys., vol. 13, no. 1, pp. 115-123, January 1972.

[16] K. Tanaka and T. Morita, "Cluster variation method and image restoration problem," Physics Letters $A$, vol. 203, no. 2-3, pp. 122-128, July 1995.

[17] M. Abramowitz and I. A. Stegun, Handbook of Mathematical Functions with Formulas, Graphs, and Mathematical Tables (Dover Publ. Inc., New York, 1965) p. 788 and p. 791.

[18] T. Morita and K. Tanaka, "Cluster ZTP in the recovery of an image," Pattern Recognition Letters, vol. 18, no. 14, pp. 1479-1493, December 1997.

[19] K. Tanaka and T. Horiguchi, "Quantum statistical-mechanical iterative method in image restoration," Trans. Inst. Electron. Inf. Commun. Eng. A, vol. J80-A, no. 12, pp. 2117-2126, December 1997 (in Japanese); Electronics and Communications in Japan, part 3, vol. 83, no. 3, pp. 84-94, 2000 (translated in English).

[20] J. Inoue, "Image restoration using quantum fluctuation," Proceedings of the 10th International Conference on Image Analysis and Processing (Venice, Italy), pp. 130-135, 1999.

[21] J. Inoue, "Application of the quantum spin glass theory to image restoration," Physical Review E, vol. 63, 2001, in press.

[22] K. Tanaka, "Theoretical study of hyperparameter estimation by maximization of marginal likelihood in image restoration by means of cluster variation method," Trans. Inst. Electron. Inf. Commun. Eng. A, vol. J83-A, no. 10, pp. 1148-1160, October 2000 (in Japanese); Electronics and Communications in Japan, part 3, vol. 85, no. 7, pp. 50-62, 2002 (translated in English). 